\title{
GENERALIZED WEIGHTED SOBOLEV SPACES AND APPLICATIONS TO SOBOLEV ORTHOGONAL POLYNOMIALS I
}

\author{
José M. Rodríguez ${ }^{*}$ Venancio Álvarez, Elena Romera*, Domingo Pestana*
}

\section{Introduction and main results.}

Weighted Sobolev spaces are an interesting topic in many fields of Mathematics. In the classical books $[\mathrm{Ku}],[\mathrm{KS}]$, we can find the point of view of Partial Differential Equations. See also [Tr] and [HKM]. (The main topic of $[\mathrm{HKM}]$ is non-linear Partial Differential Equations and its applications to quasiconformal and quasiregular maps.) We are interested in the relationship between this topic and Approximation Theory in general, and Sobolev Orthogonal Polynomials in particular.

The specific problems we want to solve are the following:

1) Given a Sobolev scalar product with general measures in $\mathbf{R}$, find hypotheses on the measures, as general as possible, so that we can define a Sobolev space whose elements are functions.

2) If a Sobolev scalar product with general measures in $\mathbf{R}$ is well defined for polynomials, what is the completion, $P^{k, 2}$, of the space of polynomials with respect to the norm associated to that scalar product? This problem has been studied in some very particular cases (see e.g. [ELW1], [EL], [ELW2]), but at this moment no general theory has been built.

3) What are the most general conditions under which the multiplication operator, $M f(x)=x f(x)$, is bounded in the space $P^{k, 2}$ ? We know by a theorem in [LPP] that the zeroes of the Sobolev orthogonal polynomials are contained in the disk $\{z:|z| \leq\|M\|\}$. The location of these zeroes allows to prove results on the asymptotic behaviour of Sobolev orthogonal polynomials (see [LP]). In the second part of this paper, [RARP], and in [R2] and [APRR], we answer the question stated also in [LP] about general conditions for $M$ to be bounded.

This last question is very close to the definition of Sobolev spaces associated to these norms, the study of their completeness and the density of $C^{\infty}$ functions. In fact, the definition and completeness of Sobolev spaces is what we study in the present paper, not only for $p=2$, but for $1 \leq p \leq \infty$. An important part of this paper concerns the development of technical tools. These tools are crucial not only here but in the papers [RARP], [R1], [R2], [R3], [APRR] and [RY]. The problems about density and about the multiplication operator are studied in these six papers.

The completeness that we study now is one of the central questions in the theory of weighted Sobolev spaces, together with the density of $C^{\infty}$ functions. In particular, when all the measures are finite, have compact support and are such that $C_{c}^{\infty}(\mathbf{R})$ is dense in a Sobolev space that is complete, then the closure of the polynomials is the whole Sobolev space. This is deduced from Bernstein's proof of Weierstrass' theorem, where the polynomials he builds approximate uniformly up to the $k$-th derivative any function in $C^{k}([a, b])$ (see e.g. [D, p.113]).

In this paper we also prove some inequalities which generalize classical results about Sobolev spaces with respect to Lebesgue measure (see Theorem 4.3).

We should remark that there exists another generalization of Sobolev spaces in the context of metric spaces (see $[\mathrm{H}],[\mathrm{M}])$. In these papers the treatment of this topic is from a different point of view.

The first part of this article is devoted to obtain a good definition of Sobolev space, $W^{k, p}\left(\mu_{0}, \ldots, \mu_{k}\right)$, where $\mu_{0}, \ldots, \mu_{k}$, are very general measures. We allow the measures $\mu_{0}, \ldots, \mu_{k}$, to be almost independent of each other. The main result that we present in this paper is Theorem 5.1, which appears in Section 5. It states very general conditions on the measures under which this Sobolev space is complete.

2000 AMS Subject Classification: 41A10, 46E35, 46G10.

* Research partially supported by a grant from DGI (BFM 2000-0206-C04-01), Spain. 
The main ingredient of the proof of this theorem is Theorem 4.3. It allows to control the $L^{\infty}$ norm (in appropriate sets) of a function and its dervatives in terms of its Sobolev norm. It is also useful by its applications in the papers [RARP], [R1], [R2], [R3], [APRR] and [RY]. Furthermore, it is important by itself, since it answers to the following main question: when the evaluation functional of $f$ (or $f^{(j)}$ ) in a point is a bounded operator in $W^{k, p}(\bar{\Omega}, \mu)$ ?

As a consequence of theorems 4.3 and 5.1, we can prove the density of the space of polynomials in these Sobolev spaces (see [RARP], [R1], [R3], [APRR] and [RY]) and the boundedness of the multiplication operator (see [RARP], [R2] and [APRR]).

In the paper, the results are numbered according to the section where they are proved. Now we present the notation we use.

Notation. In the paper, $k \geq 1$ denotes a fixed natural number; obviously $W^{0, p}(\bar{\Omega}, \mu)=L^{p}(\bar{\Omega}, \mu)$. All the measures we consider are Borel and positive. Also, all the weights are non-negative Borel measurable functions. If the measure does not appear explicitly, we mean that we are using Lebesgue measure. We allow measures $\mu_{j}$ which are not necessarily $\sigma$-finite but always assume that $d \mu_{j}=d\left(\mu_{j}\right)_{s}+w_{j} d x$, where $\left(\mu_{j}\right)_{s}$ is singular with respect to Lebesgue measure and $w_{j}$ is a Lebesgue measurable function (which can be infinite in a set of positive Lebesgue measure). We denote by supp $\nu$ the support of the measure $\nu$. If $A$ is a Borel set, $|A|, \chi_{A}, \bar{A}, \operatorname{int}(A)$ and $\# A$ denote, respectively, the Lebesgue measure, the characteristic function, the closure, the interior and the cardinality of $A$. By $f^{(j)}$ we mean the $j$-th distributional derivative of $f$. When we work in the space $W^{k, p}(\bar{\Omega}, \mu)$ we denote by $W^{k-r, p}(\bar{\Omega}, \mu)$ the space $W^{k-r, p}\left(\bar{\Omega},\left(\mu_{r}, \ldots, \mu_{k}\right)\right)$. We say that an $n$-dimensional vector satisfies a one-dimensional property if each coordinate satisfies this property. $P_{n}$ denotes the set of polynomials with degree less than or equal to $n$, and $a, b$ arbitrary real numbers with $a<b$; they are finite unless the contrary is specified. Finally, the constants in the formulae can vary from line to line and even in the same line.

The outline of the paper is as follows. Section 2 presents most of the definitions we need to state our results. We prove some useful results on Hardy inequalities and comparable norms in Section 3 . Section 4 is dedicated to some technical results; some of them are generalizations of classical results with Lebesgue measure. Finally, in Section 5 we prove the theorem on completeness.

Acknowledgements. We would like to thank F. Marcellán for suggesting us this problem and H. Pijeira for many useful references.

\section{Definitions.}

There are two standard ways to define classical Sobolev spaces $W^{k, p}(\Omega)$ (with $1 \leq p<\infty$ ) in an open subset $\Omega$ of an Euclidean space:

(1) the completion of smooth functions $C^{\infty}(\Omega)$ with the norm

$$
\|f\|_{k, p}:=\sum_{\alpha \leq k}\left\|D^{\alpha} f\right\|_{p}
$$

where $\|g\|_{p}$ denotes the $L^{p}(\Omega)$ norm of $g$ with respect to Lebesgue measure, and

(2) the functions $f$ belonging to $L^{p}(\Omega)$ such that their weak derivatives up to order $k$ belong also to $L^{p}(\Omega)$.

It is well-known that these two definitions are equivalent for $1 \leq p<\infty$ (see e.g. [A, p.52], [Ma, p.12]). However (1) and (2) coincide with the completion of $C^{\infty}\left(\mathbf{R}^{n}\right)$ only for smooth domains (see e.g. [A, p.54], [Ma, p.14]).

It is possible to define some particular weighted Sobolev spaces, where the weights considered are powers of $d(x)=\operatorname{dist}(x, K)$ with $K \subseteq \partial \Omega$, and even $h(d(x))$ with $h$ a monotone function, following the text [Ku]. If we want to define more general weighted Sobolev spaces we can use the approach in [KO]. Before we state the definition in $[\mathrm{KO}]$, let us observe that the distributional derivative of a Sobolev function is also a function belonging to $L_{l o c}^{1}(\Omega)$. In order to get the inclusion

$$
L^{p}(\Omega, u) \subseteq L_{l o c}^{1}(\Omega), \quad \text { for } 1<p<\infty,
$$


a sufficient condition, by Hölder's inequality, is that the weight $u$ satisfies $u^{-1 /(p-1)} \in L_{l o c}^{1}(\Omega)$ (see [KO, Theorem 1.5] or Lemma 3.1 below). With this fact in mind we can understand the definition in [KO]:

Given a weight $u$ in $\Omega$ let us denote by $M_{p}(u)$, for $1<p<\infty$, the closed set

$$
M_{p}(u):=\left\{x \in \Omega: \int_{\Omega \cap U(x)} u^{-1 /(p-1)}(y) d y=\infty \text { for every neighbourhood } U(x) \text { of } x\right\} .
$$

Given $w=\left(w_{\alpha}\right)_{\alpha<k}$ a vectorial weight in $\Omega$ we can define the exceptional set $B:=\cup_{\alpha}{ }_{k} M_{p}\left(w_{\alpha}\right)$ and the Sobolev space $W^{k, p}(\Omega, w)$ with weight $w$, as the set of all functions $f \in L^{p}\left(\Omega \backslash B, w_{0}\right)$ such that their weak derivatives $D^{\alpha} f$ are elements of $L^{p}\left(\Omega \backslash B, w_{\alpha}\right)$ for all $\alpha$ with $|\alpha| \leq k$.

With this definition, the weighted Sobolev space $W^{k, p}(\Omega, w)$ is a Banach space (see [KO, Section 3]).

In general, this is not true without removing the set $B$ (see some examples in [KO]). However, note that if some $w_{\alpha}$ is identically zero, then $M_{p}\left(w_{\alpha}\right)=\Omega$ and $\Omega \backslash B=\emptyset$.

But now, we want to define a more general class of Sobolev spaces appearing in the context of orthogonal polynomials. Since we are interested in orthogonal polynomials on the real line we only need to consider the case $\Omega \subseteq \mathbf{R}$. In this field it is usual to work with Sobolev spaces for which the measures $w_{j}(x) d x$ are replaced by general measures $d \mu_{j}(x)$ and some of them may have $\mu_{j}(\partial \Omega)>0$; so we consider in our definition Sobolev spaces in $\bar{\Omega}$, where $\Omega$ is an open set. Therefore, in general, these spaces do not match the definition in $[\mathrm{KO}]$.

Let us start with some preliminary definitions.

Definition 1. We say that two functions $u, v$ are comparable on the set $A$ if there are positive constants $c_{1}, c_{2}$ such that $c_{1} v(x) \leq u(x) \leq c_{2} v(x)$ for almost every $x \in A$. Since measures and norms are functions on measurable sets and vectors, respectively, we can talk about comparable measures and comparable norms. We say that two vectorial weights or vectorial measures are comparable if each component is comparable.

In what follows, the symbol $a \asymp b$ means that $a$ and $b$ are comparable for $a$ and $b$ functions, measures or norms.

Obviously, the spaces $L^{p}(A, \mu)$ and $L^{p}(A, \nu)$ are the same and have comparable norms if $\mu$ and $\nu$ are comparable on $A$. Therefore, in order to obtain results on completeness or density we can change a measure $\mu$ to any comparable measure $\nu$.

Next, we shall define a class of weights which plays an important role in our results.

Definition 2. We say that a weight $w$ belongs to $B_{p}([a, b])$ if and only if

$$
\begin{array}{ll}
w^{-1} \in L^{1 /(p-1)}([a, b]), & \text { for } 1 \leq p<\infty, \\
w^{-1} \in L^{1}([a, b]), & \text { for } p=\infty .
\end{array}
$$

Also, if $J$ is any interval we say that $w \in B_{p}(J)$ if $w \in B_{p}(I)$ for every compact interval $I \subseteq J$. We say that a weight belongs to $B_{p}(J)$, where $J$ is a union of disjoint intervals $\cup_{i \in A} J_{i}$, if it belongs to $B_{p}\left(J_{i}\right)$, for $i \in A$.

Observe that if $v \geq w$ in $J$ and $w \in B_{p}(J)$, then $v \in B_{p}(J)$.

This class contains the classical $A_{p}$ weights appearing in Harmonic Analysis (see [Mu1] or [GR]). The classes $B_{p}(\Omega)$, with $\Omega \subseteq \mathbf{R}^{n}$, and $A_{p}\left(\mathbf{R}^{n}\right)(1<p<\infty)$ have been used in other definitions of weighted Sobolev spaces in $[\mathrm{KO}]$ and $[\mathrm{K}]$ respectively.

Definition 3. We denote by $A C([a, b])$ the set of functions absolutely continuous in $[a, b]$, i.e. the functions $f \in C([a, b])$ such that $f(x)-f(a)=\int_{a}^{x} f^{\prime}(t) d t$ for all $x \in[a, b]$. If $J$ is any interval, AC $C_{\text {loc }}(J)$ denotes the set of functions absolutely continuous in every compact subinterval of $J$.

Definition 4. Let us consider $1 \leq p \leq \infty$ and a vectorial measure $\mu=\left(\mu_{0}, \ldots, \mu_{k}\right)$. For $0 \leq j \leq k$ we define the open set

$$
\Omega_{j}:=\left\{x \in \mathbf{R}: \exists \text { an open neighbourhood } V \text { of } x \text { with } w_{j} \in B_{p}(V)\right\}
$$


Observe that we always have $w_{j} \in B_{p}\left(\Omega_{j}\right)$ for any $0 \leq j \leq k$. In fact, $\Omega_{j}$ is the largest open set $U$ with $w_{j} \in B_{p}(U)$. Obviously, $\Omega_{j}$ depends on $p$ and $\mu$, although $p$ and $\mu$ do not appear explicitly in the symbol $\Omega_{j}$. Lemma 3.1 below gives that if $f^{(j)} \in L^{p}\left(\Omega_{j}, w_{j}\right)$ with $0 \leq j \leq k$, then $f^{(j)} \in L_{l o c}^{1}\left(\Omega_{j}\right)$, and therefore $f^{(j-1)} \in A C_{l o c}\left(\Omega_{j}\right)$ if $1 \leq j \leq k$.

Hypothesis. From now on we assume that $w_{j}$ is identically 0 on the complement of $\Omega_{j}$.

We need this hypothesis in order to obtain complete Sobolev spaces (see[KO] and sections 4 and 5).

Remark. This hypothesis is satisfied, for example, if we can modify $w_{j}$ in a set of zero Lebesgue measure in such a way that there exists a sequence $\alpha_{n} \searrow 0$ with $w_{j}^{-1}\left\{\left(\alpha_{n}, \infty\right]\right\}$ open for every $n$. If $w_{j}$ is lower semicontinuous, then this condition is satisfied.

Let us consider $1 \leq p \leq \infty$, an open set $\Omega \subseteq \mathbf{R}, w=\left(w_{0}, \ldots, w_{k}\right)$ a vectorial weight in $\Omega$ and $y \in \bar{\Omega}$. To obtain a greater regularity of the functions in a Sobolev space we construct a modification of the weight $w$ in a neighbourhood of $y$, using Muckenhoupt weighted version of Hardy inequality (see [Mu2], [Ma, p.44] or Section 3 below). This modified weight is equivalent in some sense to the original one (see Theorem 4.3).

Definition 5. A vectorial weight $\bar{w}=\left(\bar{w}_{0}, \ldots, \bar{w}_{k}\right)$ is a right completion of $w$ with respect to $y$, if $\bar{w}_{k}=w_{k}$ and there is an $\varepsilon>0$ such that $\bar{w}_{j}=w_{j}$ in the complement of $[y, y+\varepsilon]$ and

$$
\bar{w}_{j}(x)=w_{j}(x)+\widetilde{w}_{j}(x), \quad \text { for } x \in[y, y+\varepsilon] \text { and } 0 \leq j<k,
$$

where $\widetilde{w}_{j}$ is any weight satisfying:

i) $\widetilde{w}_{j} \in L^{1}([y, y+\varepsilon])$ if $1 \leq p<\infty$,

ii) $\widetilde{w}_{j} \in L^{\infty}([y, y+\varepsilon])$ if $p=\infty$,

iii) $\Lambda_{p}\left(\widetilde{w}_{j}, \bar{w}_{j+1}\right)<\infty$, with

$$
\begin{gathered}
\Lambda_{p}(u, v):=\sup _{y<r<y+\varepsilon}\left(\int_{y}^{r} u\right)\left\|v^{-1}\right\|_{L^{1 /(p-1)}([r, y+\varepsilon])}, \quad \text { for } 1 \leq p<\infty \\
\Lambda_{\infty}(u, v):=\underset{y<r<y+\varepsilon}{\operatorname{ess} \sup } u(r) \int_{r}^{y+\varepsilon} v^{-1}
\end{gathered}
$$

Example. It can be shown that the following construction is always a completion: we choose $\widetilde{w}_{j}:=0$ if $\bar{w}_{j+1} \notin B_{p}((y, y+\varepsilon])$; if $\bar{w}_{j+1} \in B_{p}([y, y+\varepsilon])$ we set $\widetilde{w}_{j}(x):=1$ in $[y, y+\varepsilon]$; and if $\bar{w}_{j+1} \in B_{p}((y, y+\varepsilon]) \backslash$ $B_{p}([y, y+\varepsilon])$ we take $\widetilde{w}_{j}(x):=1$ for $x \in[y+\varepsilon / 2, y+\varepsilon]$, and

$$
\begin{gathered}
\widetilde{w}_{j}(x):=\frac{d}{d x}\left\{\left(\int_{x}^{y+\varepsilon} \bar{w}_{j+1}^{-1 /(p-1)}\right)^{-p+1}\right\}=\frac{(p-1) \bar{w}_{j+1}(x)^{-1 /(p-1)}}{\left(\int_{x}^{y+\varepsilon} \bar{w}_{j+1}^{-1 /(p-1)}\right)^{p}}, \quad \text { if } 1<p<\infty, \\
\widetilde{w}_{j}(x):=\left\|\bar{w}_{j+1}^{-1}\right\|_{L^{\infty}([x, y+\varepsilon])}^{-1}+\frac{d}{d x}\left(\left\|\bar{w}_{j+1}^{-1}\right\|_{L^{\infty}([x, y+\varepsilon])}^{-1}\right), \quad \text { if } p=1, \\
\widetilde{w}_{j}(x):=\min \left\{1,\left(\int_{x}^{y+\varepsilon} \bar{w}_{j+1}^{-1}\right)^{-1}\right\}, \quad \text { if } p=\infty,
\end{gathered}
$$

for $x \in(y, y+\varepsilon / 2)$.

Remarks.

1. We can define a left completion of $w$ with respect to $y$ in a similar way.

2. If for every $0<\eta \leq \eta_{0} \leq \varepsilon$ we have $\bar{w}_{j+1} \notin B_{p}((y, y+\eta])$, then there exists some $\delta>0$ such that every $\widetilde{w}_{j}$ must be 0 almost everywhere in $(y, y+\delta)$ (where $\varepsilon$ is the constant corresponding to $\bar{w}$ ). Moreover, the constant $\delta$ depends on $\eta_{0}$ and $\bar{w}_{j+1}$, but not on $\widetilde{w}_{j}$.

3. If $\bar{w}_{j+1} \in B_{p}([y, y+\varepsilon])$, then $\Lambda_{p}\left(\widetilde{w}_{j}, \bar{w}_{j+1}\right)<\infty$ for any weight $\widetilde{w}_{j} \in L^{1}([y, y+\varepsilon])$ if $1 \leq p<\infty$ and for any bounded weight $\widetilde{w}_{j}$ if $p=\infty$. In particular, $\Lambda_{p}\left(1, \bar{w}_{j+1}\right)<\infty$.

4. If $w, v$ are two weights such that $w_{j} \geq c v_{j}$ for $j=0, \ldots, k$ and $\bar{v}$ is a right completion of $v$, then there is a right completion $\bar{w}$ of $w$, with $\bar{w}_{j} \geq c \bar{v}_{j}$ for $j=0, \ldots, k$ (it is enough to take $\widetilde{w}_{j}=\widetilde{v}_{j}$ ). Also, if 
$w, v$ are comparable weights, $\bar{v}$ is a right completion of $v$ if and only if it is comparable to a right completion $\bar{w}$ of $w$.

5. The hypotheses i) and ii) are not restrictive at all; if we are interested in the regularity of Sobolev functions we must choose weights without "big" singularities.

6. We always have $\bar{w}_{k}=w_{k}$ and $\bar{w}_{j} \geq w_{j}$ for $0 \leq j<k$.

7. If $\bar{w}$ is a right completion of $w$ with constant $\varepsilon>0$, the weight $\bar{w}^{*}=\left(\bar{w}_{0}^{*}, \ldots, \bar{w}_{k}^{*}\right)$ defined by

$$
\bar{w}_{j}^{*}(x)= \begin{cases}\bar{w}_{j}(x), & x \in[y, y+\delta], \\ w_{j}(x), & x \notin[y, y+\delta],\end{cases}
$$

for some $0<\delta<\varepsilon$, is a right completion of $w$ with constant $\delta$.

Definition 6. For $1 \leq p \leq \infty$ and $w$ a vectorial weight in $\Omega$, we say that a point $y \in \bar{\Omega}$ is right $j$-regular (respectively, left $j$-regular), if there exist $\varepsilon>0$, a right completion $\bar{w}$ (respectively, left completion) and $j<i \leq k$ such that $\bar{w}_{i} \in B_{p}([y, y+\varepsilon])$ (respectively, $B_{p}([y-\varepsilon, y])$ ). Also, we say that a point $y \in \bar{\Omega}$ is $j$-regular, if it is right and left $j$-regular.

\section{Remarks.}

1. A point $y \in \bar{\Omega}$ is right $j$-regular (respectively, left $j$-regular), if at least one of the following properties is verified:

(a) There exist $\varepsilon>0$ and $j<i \leq k$ such that $w_{i} \in B_{p}([y, y+\varepsilon])$ (respectively, $B_{p}([y-\varepsilon, y])$ ). Here we have chosen $\widetilde{w}_{j}=0$ and $\bar{w}=w$.

(b) There exist $\varepsilon>0, j<i \leq k, \alpha>0, \delta<\delta_{p}$, with $\delta_{p}:=(i-j) p-1$ if $1 \leq p<\infty$ and $\delta_{\infty}:=i-j-1$, such that

$$
w_{i}(x) \geq \alpha|x-y|^{\delta}, \quad \text { for almost every } x \in[y, y+\varepsilon]
$$

(respectively, $[y-\varepsilon, y]$ ). See Lemma 3.4 below.

2. If $y$ is right $j$-regular (respectively, left), then it is also right $i$-regular (respectively, left) for each $0 \leq i \leq j$.

3. We can take $i=j+1$ in this definition since by the third remark after Definition 5 we can choose $\bar{w}_{l}=w_{l}+1 \in B_{p}([y, y+\varepsilon])$ for $j<l<i$, if $j+1<i$.

4. If we define

$$
k_{0}:=\max \left\{0 \leq j \leq k: \exists \eta>0 \text { with } w_{j} \in B_{p}((y, y+\eta])\right\},
$$

the completion $\bar{w}$ in Definition 6 can be chosen as $\bar{w}_{j}=w_{j}$ for $k_{0} \leq j \leq k$ and $\bar{w}_{k_{0}}=w_{k_{0}} \in B_{p}((y, y+\varepsilon])$. This is an immediate consequence of remarks 2 and 7 to Definition 5 .

When we use this definition we think of a point $\{b\}$ as the union of two half-points $\left\{b^{+}\right\}$and $\left\{b^{-}\right\}$. With this convention, each one of the following sets

$$
\begin{aligned}
& (a, b) \cup(b, c) \cup\left\{b^{+}\right\}=(a, b) \cup\left[b^{+}, c\right) \neq(a, c), \\
& (a, b) \cup(b, c) \cup\left\{b^{-}\right\}=\left(a, b^{-}\right] \cup(b, c) \neq(a, c),
\end{aligned}
$$

has two connected components, and the set $(a, b) \cup(b, c) \cup\left\{b^{-}\right\} \cup\left\{b^{+}\right\}=(a, b) \cup(b, c) \cup\{b\}=(a, c)$ is connected.

We only use this convention in order to study the sets of continuity of functions: we want that if $f \in C(A)$ and $f \in C(B)$, where $A$ and $B$ are union of intervals, then $f \in C(A \cup B)$. With the usual definition of continuity in an interval, if $f \in C([a, b)) \cap C([b, c])$ then we do not have $f \in C([a, c])$. Of course, we have $f \in C([a, c])$ if and only if $f \in C\left(\left[a, b^{-}\right]\right) \cap C\left(\left[b^{+}, c\right]\right)$, where, by definition, $C\left(\left[b^{+}, c\right]\right)=C([b, c])$ and $C\left(\left[a, b^{-}\right]\right)=C([a, b])$. This idea can be formalized with a suitable topological space.

Let us introduce some more notation. We denote by $\Omega^{(j)}$ the set of $j$-regular points or half-points, i.e., $y \in \Omega^{(j)}$ if and only if $y$ is $j$-regular, we say that $y^{+} \in \Omega^{(j)}$ if and only if $y$ is right $j$-regular, and we say that $y^{-} \in \Omega^{(j)}$ if and only if $y$ is left $j$-regular. Obviously, $\Omega^{(k)}=\emptyset$ and $\Omega_{j+1} \cup \cdots \cup \Omega_{k} \subseteq \Omega^{(j)}$. Observe that $\Omega^{(j)}$ depends on $p$ (see Definition 6). 
Remark. If $0 \leq j<k$ and $I$ is an interval, $I \subseteq \Omega^{(j)}$, then the set $I \backslash\left(\Omega_{j+1} \cup \cdots \cup \Omega_{k}\right)$ is discrete. If $y^{+} \in I \backslash\left(\Omega_{j+1} \cup \cdots \cup \Omega_{k}\right)$, there exist $\varepsilon>0$, a right completion $\bar{w}$ and $j<i \leq k$ with $\bar{w}_{i} \in B_{p}([y, y+\varepsilon])$. Then there exist $\delta>0$ and $i \leq l \leq k$ with $w_{l} \in B_{p}((y, y+\delta])$ and consequently $(y, y+\delta) \subseteq \Omega_{j+1} \cup \cdots \cup \Omega_{k}$ (see the second remark to Definition 5). Obviously the same is true for $y^{-}$.

Definition 7. We say that a function $h$ belongs to the class $A C_{l o c}\left(\Omega^{(j)}\right)$ if $h \in A C_{l o c}(I)$ for every connected component $I$ of $\Omega^{(j)}$.

Definition 8. We say that the vectorial measure $\mu=\left(\mu_{0}, \ldots, \mu_{k}\right)$ is p-admissible if $\left(\mu_{j}\right)_{s}\left(\mathbf{R} \backslash \Omega^{(j)}\right)=0$, for $1 \leq j<k$, and $\left(\mu_{k}\right)_{s} \equiv 0$. We say that a p-admissible vectorial measure, $\mu$, is strongly $p$-admissible if $\operatorname{supp}\left(\mu_{j}\right)_{s} \subseteq \Omega^{(j)}$, for $1 \leq j<k$.

We use the letter $p$ in $p$-admissible in order to emphasize the dependence on $p$ (recall that $\Omega^{(j)}$ depends on $p$ ).

\section{Remarks.}

1. Observe that there is not any restriction on $\operatorname{supp}\left(\mu_{0}\right)_{s}$.

2. Every absolutely continuous measure is $p$-admissible and even strongly $p$-admissible.

3. We want to remark that this definition of $p$-admissibility does not coincide with the one in [HKM].

Definition 9. (Sobolev space in the closure of an open set.) Let us consider $1 \leq p \leq \infty$, an open set $\Omega \subseteq \mathbf{R}$ and a p-admissible vectorial measure $\mu=\left(\mu_{0}, \ldots, \mu_{k}\right)$ in $\bar{\Omega}$. We define the Sobolev space $W^{k, p}(\bar{\Omega}, \mu)$ as the space of equivalence classes of

$$
\begin{gathered}
V^{k, p}(\bar{\Omega}, \mu):=\left\{f: \bar{\Omega} \rightarrow \mathbf{C} / f^{(j)} \in A C_{l o c}\left(\Omega^{(j)}\right) \text { for } j=0,1, \ldots, k-1\right. \text { and } \\
\left.\left\|f^{(j)}\right\|_{L^{p}\left(\bar{\Omega}, \mu_{j}\right)}<\infty \text { for } j=0,1, \ldots, k\right\},
\end{gathered}
$$

with respect to the seminorms

$$
\begin{gathered}
\|f\|_{W^{k, p}(\bar{\Omega}, \mu)}:=\left(\sum_{j=0}^{k}\left\|f^{(j)}\right\|_{L^{p}\left(\bar{\Omega}, \mu_{j}\right)}^{p}\right)^{1 / p}, \quad \text { for } 1 \leq p<\infty, \\
\|f\|_{W^{k, \infty}(\bar{\Omega}, \mu)}:=\max _{0 \leq j \leq k}\left\|f^{(j)}\right\|_{L^{\infty}\left(\bar{\Omega}, \mu_{j}\right)} .
\end{gathered}
$$

Here

$$
\|g\|_{L^{\infty}\left(\Omega, \mu_{j}\right)}:=\max \left\{\operatorname{essup}_{x \in \Omega}\left|g(x) w_{j}(x)\right| \sup _{x \in \operatorname{supp}\left(\mu_{j}\right)_{s}}|g(x)|\right\},
$$

where ess sup refers to Lebesgue measure, and we assume the usual convention $\sup \emptyset=-\infty$.

Remarks.

1. This definition is natural since when the $\left(\mu_{j}\right)_{s}$-measure of the set where $\left|f^{(j)}\right|$ is not continuous is positive, the integral $\int\left|f^{(j)}\right|^{p} d\left(\mu_{j}\right)_{s}$ does not make sense.

2. If we consider Sobolev spaces with real valued functions, every result in this paper also holds.

An example of Sobolev space as we have just defined is the following: $W^{2,2}([0,6], \mu)$, where

$$
\|f\|_{W^{2,2}([0,6], \mu)}^{2}=\int_{4}^{6}|f|^{2}+|f(6)|^{2}+\int_{0}^{1}\left|f^{\prime}\right|^{2} \sqrt{x}+\int_{3}^{5}\left|f^{\prime}\right|^{2} \sqrt{x-3}+\left|f^{\prime}(1)\right|^{2}+\int_{1}^{3}\left|f^{\prime \prime}\right|^{2}(3-x) .
$$

In this example, $w_{0} \in B_{2}([4,6]), w_{1} \in B_{2}([0,1] \cup[3,5]), w_{2} \in B_{2}([1,3))$, and consequently $\Omega_{0}=(4,6)$, $\Omega_{1}=(0,1) \cup(3,5)$ and $\Omega_{2}=(1,3)$; therefore, $\Omega^{(1)}=[1,3)$ and $\Omega^{(0)}=[0,5]$. Observe that 3 is right 0 -regular since $w_{1} \in B_{2}([3,5])$, and that 3 is left 0 -regular since we can take $\widetilde{w}_{1}=1$ in $[1,3]$. If we add $\delta_{a}$ to $\mu_{1}$, we obtain a $p$-admissible measure (and the Sobolev space is well defined) if and only if $a \in[1,3$ ). We can add $\delta_{a}$ to $\mu_{0}$ for any $a \in \mathbf{R}$, and we can not add $\delta_{a}$ to $\mu_{2}$ for any $a \in \mathbf{R}$. Obviously, in this definition $f^{\prime}(1)$ stands for $f^{\prime}\left(1^{+}\right)$, since $f^{\prime} \in A C_{l o c}([1,3))$. 


\section{Applications of Hardy and Muckenhoupt inequalities.}

First of all we recall the classical results.

Hardy inequality. ([HLP]) If $1 \leq p<\infty$ and $\delta>p-1$, then

$$
\int_{0}^{\infty}\left|\int_{x}^{\infty} g(t) d t\right|^{p} x^{\delta-p} d x \leq\left(\frac{p}{\delta-p+1}\right)^{p} \int_{0}^{\infty}|g(x)|^{p} x^{\delta} d x
$$

for any measurable function $g$ in $(0, \infty)$.

First Muckenhoupt inequality. ([Mu2], [Ma, p.44]) Let us consider $1 \leq p<\infty$ and $\mu_{0}, \mu_{1}$ measures in $(0, \infty)$ with $w_{1}:=d \mu_{1} / d x$. Then there exists a positive constant $c$ such that

$$
\left\|\int_{x}^{\infty} g(t) d t\right\|_{L^{p}\left((0, \infty), \mu_{0}\right)} \leq c\|g\|_{L^{p}\left((0, \infty), \mu_{1}\right)}
$$

for any measurable function $g$ in $(0, \infty)$, if and only if

$$
\sup _{r>0} \mu_{0}((0, r])\left\|w_{1}^{-1}\right\|_{L^{1 /(p-1)}([r, \infty))}<\infty
$$

The same proof of the first Muckenhoupt inequality with the real numbers $a, b$ instead of 0 , $\infty$, gives the following result.

Second Muckenhoupt inequality. Let us consider $1 \leq p<\infty$ and $\mu_{0}, \mu_{1}$ measures in $(a, b]$ with $w_{1}:=$ $d \mu_{1} / d x$. Then there exists a positive constant $c$ such that

$$
\left\|\int_{x}^{b} g(t) d t\right\|_{L^{p}\left((a, b], \mu_{0}\right)} \leq c\|g\|_{L^{p}\left((a, b], \mu_{1}\right)}
$$

for any measurable function $g$ in $(a, b]$, if and only if

$$
\Lambda_{p}\left(\mu_{0}, \mu_{1}\right):=\sup _{a<r<b} \mu_{0}((a, r])\left\|w_{1}^{-1}\right\|_{L^{1 /(p-1)}([r, b))}<\infty
$$

There are previous results to these inequalities due to Talenti [T] and Tomaselli [To]. Also, there is a contemporary work of the Muckenhoupt inequalities, by Chisholm and Everitt [CE], revised in the subsequent paper with Littlejohn [CEL].

In fact, the Hardy and Muckenhoupt inequalities are more general, but these versions are good enough for us. Let us prove now the first lemma we need for our estimates.

Lemma 3.1. Let us consider $1 \leq p \leq \infty$ and $w \in B_{p}((a, b))$. For any compact interval $I \subseteq(a, b)$, there is a positive constant $c_{1}$, which only depends on $p, w$ and $I$, such that

$$
\|g\|_{L^{1}(I)} \leq c_{1}\|g\|_{L^{p}(I, w)} \leq c_{1}\|g\|_{L^{p}([a, b], w)}, \quad \text { for any } g \in L^{p}([a, b], w) .
$$

If furthermore $w \in B_{p}([a, b])$, there is a positive constant $c_{2}$, which only depends on $p$ and $w$ such that

$$
\|g\|_{L^{1}([a, b])} \leq c_{2}\|g\|_{L^{p}([a, b], w)}, \quad \text { for any } g \in L^{p}([a, b], w) .
$$

Consequently, if $w \in B_{p}([a, b])$ and $f^{\prime} \in L^{p}([a, b], w)$, then $f \in A C([a, b])$.

Remark. The hypothesis $w \in B_{p}([a, b])$ is necessary if we want to have $g \in L^{1}([a, b])$ as shows the following example. For $1<p<\infty$ let us consider $w(x)=x^{p-1}$ in $[0,1 / 2]$. (Observe that $x^{\alpha} \in B_{p}([0,1 / 2])$ if and only if $\alpha<p-1$.) The function $g(x)=-1 /(x \log x)$ belongs to $L^{p}([0,1 / 2], w)$ but it is not integrable in [0,1/2]. It is easy to construct similar examples in the cases $p=1$ and $p=\infty$. 
Proof. Let us fix any compact interval $I \subseteq(a, b)$.

Let us begin with the case $1 \leq p<\infty$. Using Hölder's inequality

$$
\int_{I}|g|=\int_{I}|g| w^{1 / p} w^{-1 / p} \leq\|g\|_{L^{p}(I, w)}\left\|w^{-1}\right\|_{L^{1 /(p-1)}(I)}^{1 / p},
$$

which is the first part of the lemma with constant $c_{1}=\left\|w^{-1}\right\|_{L^{1 /(p-1)}(I)}^{1 / p}$.

Next, set $p=\infty$. Observe that $|g(x)| \leq\|g\|_{L^{\infty}(I, w)} w(x)^{-1}$ for almost every $x \in I$, and consequently

$$
\int_{I}|g| \leq\|g\|_{L^{\infty}(I, w)} \int_{I} w^{-1}
$$

For any case $1 \leq p \leq \infty$, if $w \in B_{p}([a, b])$ we obtain the same result changing $I$ by $[a, b]$.

The last conclusion of Lemma 3.1 is an immediate consequence of these estimates.

The following result generalizes the second Muckenhoupt inequality.

Lemma 3.2. Let us consider $1 \leq p \leq \infty, t>0$ and $\mu_{0}, \mu_{1}$ measures in $(a, b]$ with $a+t \leq b, w_{0}:=d \mu_{0} / d x$ and $w_{1}:=d \mu_{1} / d x$, satisfying if $a+t<b$ : (i) $w_{1} \in B_{p}([a+t, b])$; (ii) $\mu_{0}((a, b])<\infty$ if $1 \leq p<\infty$, (iii) $w_{0} \in L^{\infty}([a+t, b])$ if $p=\infty$. Let us assume that $\Lambda_{p}^{\prime}\left(\mu_{0}, \mu_{1}\right)<\infty$, where

$$
\begin{aligned}
& \Lambda_{p}^{\prime}\left(\mu_{0}, \mu_{1}\right):=\sup _{a<r<a+t} \mu_{0}((a, r])\left\|w_{1}^{-1}\right\|_{L^{1 /(p-1)}([r, b])}, \quad \text { for } 1 \leq p<\infty, \\
& \Lambda_{\infty}^{\prime}\left(\mu_{0}, \mu_{1}\right):= \begin{cases}\operatorname{ess} \sup _{a<r<a+t} w_{0}(r) \int_{r}^{b} w_{1}^{-1}, & \text { if }\left(\mu_{0}\right)_{s}((a, b])=0, \\
\max \left\{\operatorname{ess} \sup _{a<r<a+t} w_{0}(r) \int_{r}^{b} w_{1}^{-1}, \int_{\alpha}^{b} w_{1}^{-1}\right\}, & \text { if }\left(\mu_{0}\right)_{s}((a, b])>0,\end{cases}
\end{aligned}
$$

where $\alpha:=\min \left(\operatorname{supp}\left(\mu_{0}\right)_{s}\right)$. Then $\Lambda_{p}\left(\mu_{0}, \mu_{1}\right)<\infty$ and this implies that there exists a positive constant $c$ such that

$$
\left\|\int_{x}^{b} g(s) d s\right\|_{L^{p}\left((a, b], \mu_{0}\right)} \leq c\|g\|_{L^{p}\left((a, b], \mu_{1}\right)}
$$

for any measurable function $g$ in $(a, b]$, where $\Lambda_{p}\left(\mu_{0}, \mu_{1}\right)$ is defined changing $a+t$ by $b$ in the definition of $\Lambda_{p}^{\prime}\left(\mu_{0}, \mu_{1}\right)$.

Proof. We omit the proof for $1 \leq p<\infty$, which is only a computation that uses Muckenhoupt inequality. If $p=\infty$ and $a+t<b$, we have that $\Lambda_{\infty}\left(\mu_{0}, \mu_{1}\right)<\infty$, since $\Lambda_{\infty}^{\prime}\left(\mu_{0}, \mu_{1}\right)<\infty$ and

$$
\underset{a+t \leq r<b}{\operatorname{ess} \sup _{0}} w_{0}(r) \int_{r}^{b} w_{1}^{-1} \leq\left\|w_{0}\right\|_{L^{\infty}([a+t, b])} \int_{a+t}^{b} w_{1}^{-1}<\infty,
$$

since $w_{1} \in B_{\infty}([a+t, b])$ and $w_{0} \in L^{\infty}([a+t, b])$ because $a+t<b$.

Then we have

$$
\left|w_{0}(r) \int_{r}^{b} g(s) d s\right| \leq w_{0}(r) \int_{r}^{b}|g(s)| w_{1}(s) w_{1}(s)^{-1} d s \leq w_{0}(r) \int_{r}^{b} w_{1}(s)^{-1} d s\|g\|_{L^{\infty}\left((a, b], w_{1}\right)},
$$

and therefore

$$
\underset{a<r<b}{\operatorname{ess} \sup }\left|w_{0}(r) \int_{r}^{b} g(s) d s\right| \leq \Lambda_{\infty}\left(\mu_{0}, \mu_{1}\right)\|g\|_{L^{\infty}\left((a, b], w_{1}\right)},
$$

for any measurable function $g$ in $(a, b]$. The same argument without $w_{0}$ gives

$$
\sup _{r \in \operatorname{supp}\left(\mu_{0}\right)_{s}}\left|\int_{r}^{b} g(s) d s\right| \leq \sup _{\alpha \leq r \leq b}\left|\int_{r}^{b} g(s) d s\right| \leq \int_{\alpha}^{b} w_{1}(s)^{-1} d s\|g\|_{L^{\infty}\left((a, b], w_{1}\right)},
$$

for any measurable function $g$ in $(a, b]$. These inequalities give the case $p=\infty$.

The following lemma allows us to bound the norm in $W^{k, p}([a, b], \bar{w})$. 
Lemma 3.3. Let $1 \leq p \leq \infty$ and let $w=\left(w_{0}, \ldots, w_{k}\right)$ be a vectorial weight on $(a, b)$, with $w_{k_{0}} \in B_{p}((a, b])$ for some $0<k_{0} \leq k$. If we construct a right completion $\bar{w}$ of $w$ with respect to the point a taking $\varepsilon=b-a$, and $\bar{w}_{j}=w_{j}$ for $k_{0} \leq j \leq k$, then there exist positive constants $c_{j}$ such that

$$
c_{j}\left\|g^{(j)}\right\|_{L^{p}\left([a, b], \bar{w}_{j}\right)} \leq \sum_{i=j}^{k_{0}}\left\|g^{(i)}\right\|_{L^{p}\left([a, b], w_{i}\right)}+\sum_{i=j}^{k_{0}-1}\left|g^{(i)}(b)\right|,
$$

for all $0 \leq j<k_{0}$ and $g \in V^{k, p}([a, b], w)$. In particular, there is a positive constant c such that

$$
c\|g\|_{W^{k, p}([a, b], \bar{w})} \leq\|g\|_{W^{k, p}([a, b], w)}+\sum_{j=0}^{k_{0}-1}\left|g^{(j)}(b)\right|, \quad \text { for all } g \in V^{k, p}([a, b], w) .
$$

Proof. The fact $\bar{w}_{j}=w_{j}$ for $k_{0} \leq j \leq k$ and the first inequality give the second one. Then we only need to prove the first inequality. Lemma 3.2 (with $a+t=b$ ) gives

$$
c\left\|g^{(j)}(x)-g^{(j)}(b)\right\|_{L^{p}\left([a, b], \tilde{w}_{j}\right)} \leq\left\|g^{(j+1)}\right\|_{L^{p}\left([a, b], \bar{w}_{j+1}\right)},
$$

for $0 \leq j<k_{0}$. Then we have

$$
c\left\|g^{(j)}\right\|_{L^{p}\left([a, b], \tilde{w}_{j}\right)} \leq\left\|g^{(j+1)}\right\|_{L^{p}\left([a, b], \bar{w}_{j+1}\right)}+\left|g^{(j)}(b)\right|,
$$

since $\widetilde{w}_{j} \in L^{1}([a, b])$ if $1 \leq p<\infty$ and $\widetilde{w}_{j} \in L^{\infty}([a, b])$ if $p=\infty$. This inequality now gives

$$
c\left\|g^{(j)}\right\|_{L^{p}\left([a, b], \bar{w}_{j}\right)} \leq\left\|g^{(j)}\right\|_{L^{p}\left([a, b], w_{j}\right)}+\left\|g^{(j+1)}\right\|_{L^{p}\left([a, b], \bar{w}_{j+1}\right)}+\left|g^{(j)}(b)\right|,
$$

for $0 \leq j<k_{0}$. This fact and the fact that $\bar{w}_{k_{0}}=w_{k_{0}}$ prove the first inequality.

Lemma 3.4. Let $1 \leq p \leq \infty$ and let $w=\left(w_{0}, \ldots, w_{k}\right)$ be a vectorial weight on $(a, b)$, with $w_{k}(x) \geq \alpha(x-a)^{\delta}$ for almost every $x \in(a, \bar{b})$, where $\alpha>0$ and $\delta<\delta_{p}$, with $\delta_{p}:=k p-1$ if $1 \leq p<\infty$ and $\delta_{\infty}:=k-1$. Then there exist a right completion $\bar{w}$ and a positive constant $c$ such that $\bar{w}_{1} \in B_{p}([a, b])$ and

$$
c\|g\|_{L^{\infty}([a, b])} \leq\|g\|_{W^{k, p}([a, b], w)}+\sum_{j=0}^{k-1}\left|g^{(j)}(b)\right|, \quad \text { for all } g \in V^{k, p}([a, b], w) .
$$

Proof. By Lemmas 3.1 and 3.3 it is enough to prove $\bar{w}_{1} \in B_{p}([a, b])$.

Changing $\delta$ by a slightly greater number, if it is necessary, we can suppose that $(\delta+1) / p$ is not an integer if $1 \leq p<\infty$ and $\delta$ is not an integer if $p=\infty$.

Let us consider first the case $1 \leq p<\infty$. We prove now by reverse induction on $j$ that there is a weight $\bar{w}$ with $\bar{w}_{j}(x) \geq \alpha_{j}(x-a)^{\bar{\delta}-(k-j) p}$ for $x \in(a, b)$ if $\delta>(k-j) p-1$. If we take $\widetilde{w}_{j}(x)=$ $(\delta-(k-j) p+1)(x-a)^{\delta-(k-j) p}$, we obtain

$$
\int_{a}^{x} \widetilde{w}_{j}=(x-a)^{\delta-(k-j) p+1}
$$

if $\delta>(k-j) p-1$.

If $1<p<\infty$,

$$
\int_{x}^{b} \bar{w}_{j+1}^{-1 /(p-1)} \leq c(x-a)^{(-\delta+(k-j) p-1) /(p-1)}
$$

If $p=1$,

$$
\left\|\bar{w}_{j+1}^{-1}\right\|_{L^{\infty}([x, b])} \leq c(x-a)^{-\delta+k-j-1} .
$$

Then, if $1 \leq p<\infty$, we have $\Lambda_{p}\left(\widetilde{w}_{j}, \bar{w}_{j+1}\right)<\infty$ and $\bar{w}_{j}(x) \geq \alpha_{j}(x-a)^{\delta-(k-j) p}$ for $x \in(a, b)$ if $\delta>(k-j) p-1$. 
We define $k_{\delta}:=k-[(\delta+1) / p]$, where $[t]$ denotes the greatest integer which is less or equal than $t$. Then we have $\left(k-k_{\delta}\right) p-1<\delta<\left(k-k_{\delta}\right) p+p-1$ and $\bar{w}_{k_{\delta}}(x) \geq \alpha_{k_{\delta}}(x-a)^{\delta-\left(k-k_{\delta}\right) p}$. We also have $\bar{w}_{k_{\delta}} \in B_{p}([a, b])$ since $\delta-\left(k-k_{\delta}\right) p<p-1$. To finish the proof of this case it is enough to see that $k_{\delta} \geq 1$. If $k_{\delta} \leq 0$ then $\delta>\left(k-k_{\delta}\right) p-1 \geq k p-1$ which contradicts the hypothesis $\delta<\delta_{p}$.

Finally, if $p=\infty$, the proof is similar with $\widetilde{w}_{j}(x)=(x-a)^{\delta-k+j}$ and $k_{\delta}:=k-[\delta+1]$.

\section{Technical results.}

The heart of this chapter is Theorem 4.3; in order to prove it, we obtain several results which are particular cases.

Theorem 4.1. Let us consider $1 \leq p \leq \infty$ and a measure $\mu_{0}$ on $[a, b]$ such that supp $\mu_{0}$ has at least $k$ points. Let $w_{k}$ be a weight in $B_{p}([a, b])$. Then

(1) There exists a positive constant $c_{1}$ such that

$$
c_{1}\left\|g^{(k-1)}\right\|_{L^{\infty}([a, b])} \leq\|g\|_{L^{p}\left([a, b], \mu_{0}\right)}+\left\|g^{(k)}\right\|_{L^{p}\left([a, b], w_{k}\right)}, \quad \text { for all } g \text { with } g^{(k-1)} \in A C([a, b]) .
$$

(2) There exists a positive constant $c_{2}$ such that

$$
c_{2} \sum_{j=0}^{k-1}\left\|g^{(j)}\right\|_{L^{\infty}([a, b])} \leq\|g\|_{L^{p}\left([a, b], \mu_{0}\right)}+\left\|g^{(k)}\right\|_{L^{p}\left([a, b], w_{k}\right)}, \quad \text { for all } g \text { with } g^{(k-1)} \in A C([a, b]) .
$$

(3) If $k \geq 2$, let $\mu_{1}, \ldots, \mu_{k-1}$ be finite measures in $[a, b]$. If $1 \leq p<\infty$, there exists a positive constant $c_{3}$ such that

$$
c_{3} \sum_{j=0}^{k-1}\left\|g^{(j)}\right\|_{L^{p}\left([a, b], \mu_{j}\right)} \leq\|g\|_{L^{p}\left([a, b], \mu_{0}\right)}+\left\|g^{(k)}\right\|_{L^{p}\left([a, b], w_{k}\right)}, \quad \text { for all } g \text { with } g^{(k-1)} \in A C([a, b]) .
$$

Remark. In fact, the proof shows that $\left\|g^{(k)}\right\|_{L^{p}\left([a, b], w_{k}\right)}$ can be replaced by $\left\|g^{(k)}\right\|_{L^{1}([a, b])}$ in the right hand side of (1), (2) and (3).

Proof. We only have to prove (1). An induction argument gives (2) if (1) is true, since

$$
\left\|g^{(j)}\right\|_{L^{p}([a, b])} \leq c\left\|g^{(j)}\right\|_{L^{\infty}([a, b])},
$$

and 1 is a weight in $B_{p}([a, b])$. The third inequality is an immediate consequence of the second one and the finiteness of $\mu_{1}, \ldots, \mu_{k-1}$.

Without loss of generality we can assume that the functions are real valued, since we can consider the real and imaginary parts. By hypothesis, there exist $I_{1}, I_{2}, \ldots, I_{k}$ pairwise disjoint closed connected subsets of $[a, b]$ such that $\mu_{0}\left(I_{i}\right)>0, i=1,2, \ldots, k$. It is possible to choose each $I_{i}$ small enough in order to have either (i) $0<\mu_{0}\left(I_{i}\right)<\infty$, or (ii) $\mu_{0}\left(I_{i}\right)=\infty$ and $\mu_{0}(I)=\infty$ for every closed interval $I \subseteq I_{i}$.

Let $x_{i} \in I_{i}$. There exists $x_{0} \in(a, b)$ such that

$$
\frac{g^{(k-1)}\left(x_{0}\right)}{(k-1) !}=g\left[x_{1}, \ldots, x_{k}\right]
$$

(see [D, p.65]) where the differences $g\left[x_{1}, \ldots, x_{k}\right]$ are defined recursively by

$$
g\left[x_{j}\right]=g\left(x_{j}\right), \quad g\left[x_{i}, x_{j}\right]=\frac{g\left[x_{i}\right]-g\left[x_{j}\right]}{x_{i}-x_{j}}, \quad g\left[x_{1}, \ldots, x_{k}\right]=\frac{g\left[x_{1}, \ldots, x_{k-1}\right]-g\left[x_{2}, \ldots, x_{k}\right]}{x_{1}-x_{k}} .
$$


Consider $\varepsilon:=1$ if $k=1$ and $\varepsilon:=\min _{i \neq j} \operatorname{dist}\left(I_{i}, I_{j}\right)$ if $k \geq 2$. A standard induction argument gives

$$
\left|g\left[x_{1}, \ldots, x_{k}\right]\right| \leq \varepsilon^{-k}\left(\left|g\left(x_{1}\right)\right|+\left(\begin{array}{c}
k-1 \\
1
\end{array}\right)\left|g\left(x_{2}\right)\right|+\cdots+\left(\begin{array}{c}
k-1 \\
k-2
\end{array}\right)\left|g\left(x_{k-1}\right)\right|+\left|g\left(x_{k}\right)\right|\right)
$$

and so, for some positive constant $c_{3}$

$$
\left|g^{(k-1)}\left(x_{0}\right)\right| \leq c_{3} \sum_{i=1}^{k}\left|g\left(x_{i}\right)\right|
$$

If $x \in[a, b]$, Lemma 3.1 gives

$$
\left|g^{(k-1)}(x)\right| \leq\left|g^{(k-1)}\left(x_{0}\right)\right|+\int_{a}^{b}\left|g^{(k)}\right| \leq c_{3} \sum_{i=1}^{k}\left|g\left(x_{i}\right)\right|+c_{4}\left\|g^{(k)}\right\|_{L^{p}\left([a, b], w_{k}\right)} .
$$

If $1 \leq p<\infty$, let us denote by $J_{1}$ (respectively, $J_{2}$ ) the set of indices $1 \leq i \leq k$ with $\mu_{0}\left(I_{i}\right)<\infty$ (respectively, $\mu_{0}\left(I_{i}\right)=\infty$ ). If $i \in J_{2}$ we can assume that $g\left(x_{i}\right)=0$ (otherwise $\|g\|_{L^{p}\left(I_{i}, \mu_{0}\right)}=\infty$ since $g \in C\left(I_{i}\right)$ and then (1) is obviously true). Consequently, we have

$$
\left|g^{(k-1)}(x)\right| \leq c_{3} \sum_{i \in J_{1}}\left|g\left(x_{i}\right)\right|+c_{4}\left\|g^{(k)}\right\|_{L^{p}\left([a, b], w_{k}\right)} .
$$

We obtain that

$$
\left|g^{(k-1)}(x)\right|^{p} \leq c_{5}\left(\sum_{i \in J_{1}}\left|g\left(x_{i}\right)\right|^{p}+\int_{a}^{b}\left|g^{(k)}\right|^{p} w_{k}\right), \quad \text { for all } x \in[a, b], x_{i} \in I_{i} .
$$

Since $0<\mu_{0}\left(I_{i}\right)<\infty$ for $i \in J_{1}$, we can integrate in each $x_{i} \in I_{i}$ with respect to $\mu_{0}$ to obtain

$$
\left|g^{(k-1)}(x)\right|^{p} \leq c_{6}\left(\int_{a}^{b}|g|^{p} d \mu_{0}+\int_{a}^{b}\left|g^{(k)}\right|^{p} w_{k}\right), \quad \text { for all } x \in[a, b] .
$$

Therefore, we have obtained

$$
c_{1}\left\|g^{(k-1)}\right\|_{L^{\infty}([a, b])} \leq\|g\|_{L^{p}\left([a, b], \mu_{0}\right)}+\left\|g^{(k)}\right\|_{L^{p}\left([a, b], w_{k}\right)}
$$

for all $g$ with $g^{(k-1)} \in A C([a, b])$.

We now deal with the case $p=\infty$. Recall that in this case we also have (4.1) for all $x \in[a, b]$ and $x_{i} \in I_{i}$. Assume that $i$ satisfies $\left(\mu_{0}\right)_{s}\left(I_{i}\right)>0$. Then we have that $\left|g\left(x_{i}\right)\right| \leq\|g\|_{L^{\infty}\left([a, b], \mu_{0}\right)}$ if $x_{i} \in \operatorname{supp}\left(\mu_{0}\right)_{s}$.

If $\left(\mu_{0}\right)_{s}\left(I_{i}\right)=0$ for some $I_{i}$, then $d \mu_{0}(x)=w_{0}(x) d x$ in $I_{i}$ and there exists a positive number $t_{i}$ such that

$$
\int_{\left\{w_{0} \geq t_{i}\right\} \cap I_{i}} w_{0}(x) d x>0,
$$

since $\mu_{0}\left(I_{i}\right)>0$. Therefore

$$
\left|g\left(x_{i}\right)\right| \leq t_{i}^{-1}\left|g\left(x_{i}\right)\right| w_{0}\left(x_{i}\right) \leq t_{i}^{-1}\|g\|_{L^{\infty}\left([a, b], \mu_{0}\right)},
$$

for almost every $x_{i} \in I_{i} \cap\left\{w_{0} \geq t_{i}\right\}$ with respect to Lebesgue measure. As we only have a finite number of $I_{i}$, these inequalities and (4.1) finish the proof for the case $p=\infty$.

Lemma 4.1. Let us consider $x_{1}, \ldots, x_{k}$ with $x_{j} \in\left[\alpha_{j}, \beta_{j}\right]$ where $\beta_{j}<\alpha_{j+1}$ for every $1 \leq j \leq k-1$, and $f \in C^{k-1}\left(\left(\alpha_{1}, \beta_{k}\right]\right) \cap C\left(\left[\alpha_{1}, \beta_{k}\right]\right)$ with real values. Then, there exists $x_{0}=x_{0}\left(x_{1}, \ldots, x_{k}\right)$ for which

$$
\frac{f^{(k-1)}\left(x_{0}\right)}{(k-1) !}=f\left[x_{1}, \ldots, x_{k}\right]
$$

with the property $x_{0}\left(x_{1}, \ldots, x_{k}\right) \geq \alpha_{1}+\delta$ for some $\delta>0$ which is independent of $x_{1}, \ldots, x_{k}$. 
Proof. Let us define the function $h\left(x_{1}, \ldots, x_{k}\right):=(k-1) ! f\left[x_{1}, \ldots, x_{k}\right]$ and the set $T:=h\left(\left[\alpha_{1}, \beta_{1}\right] \times\right.$ $\left.\left[\alpha_{2}, \beta_{2}\right] \times \cdots \times\left[\alpha_{k}, \beta_{k}\right]\right) . T$ is compact since $h$ is continuous.

Also, let us consider for $t \in T$

$$
\xi(t):=\max \left(\left(f^{(k-1)}\right)^{-1}(t)\right) \subseteq\left(\alpha_{1}, \beta_{k}\right] .
$$

Note that in the proof of Theorem 4.1 above we saw that $\left(f^{(k-1)}\right)^{-1}(t) \neq \emptyset$ for any $t \in T$, and then $T \subseteq f^{(k-1)}\left(\left(\alpha_{1}, \beta_{k}\right)\right)$.

We can define

$$
\xi_{0}:=\inf \{\xi(t): t \in T\} .
$$

We only need to prove $\xi_{0}>\alpha_{1}$. We have $\xi_{0} \in\left[\alpha_{1}, \beta_{k}\right]$ and we can find a sequence $\left\{\xi\left(t_{n}\right)\right\}_{n=1}^{\infty}$ whose limit is $\xi_{0}$ with $\left\{t_{n}\right\}_{n=1}^{\infty} \subseteq T$. Without loss of generality we can assume that $t_{n} \rightarrow t_{0}$ for some $t_{0} \in T$ since $T$ is compact, and therefore

$$
f^{(k-1)}\left(\xi\left(t_{n}\right)\right)=t_{n} \longrightarrow t_{0}=f^{(k-1)}\left(\xi\left(t_{0}\right)\right), \quad \text { as } n \rightarrow \infty .
$$

Now, observe that $f^{(k-1)}$ is continuous on $\left[\left(\xi\left(t_{0}\right)+\alpha_{1}\right) / 2, \beta_{k}\right]$, since $\xi\left(t_{0}\right)>\alpha_{1}$. Then there exist preimages (by $f^{(k-1)}$ ) of all the points in $T$ close to $f^{(k-1)}\left(\xi\left(t_{0}\right)\right)$ as close as we wish to $\xi\left(t_{0}\right)$. Therefore, for $n$ large enough $\xi\left(t_{n}\right) \geq\left(\xi\left(t_{0}\right)+\alpha_{1}\right) / 2$. Hence we must have $\xi_{0} \geq\left(\xi\left(t_{0}\right)+\alpha_{1}\right) / 2>\alpha_{1}$.

Theorem 4.2. Let us consider $1 \leq p \leq \infty$ and a $p$-admissible vectorial measure $\mu$ in $[a, b]$ such that $(a, b)^{(0)} \cap \operatorname{supp} \mu_{0}$ has at least $k$ points and $w_{k} \in B_{p}((a, b])$. For $0 \leq j<k$ and fixed $0<\delta<b-a$ let us define $a_{j}:=0$ if $a$ is right $j$-regular and $a_{j}:=1$ otherwise. Then, there exists a positive constant $c=c(\delta)$ such that

$$
c \sum_{j=0}^{k-1}\left\|g^{(j)}\right\|_{L^{\infty}\left(\left[a+\delta a_{j}, b\right]\right)} \leq\|g\|_{W^{k, p}([a, b], \mu)}, \quad \text { for all } g \in V^{k, p}([a, b], \mu) .
$$

Proof. Without loss of generality we can assume that the functions are real valued. For each $\delta_{k-1}>0$ small enough there exists a positive constant $c_{k-1}=c_{k-1}\left(\delta_{k-1}\right)$ such that

$$
c_{k-1}\left\|g^{(k-1)}\right\|_{L^{\infty}\left(\left[a+\delta_{k-1}, b\right]\right)} \leq\|g\|_{L^{p}\left([a, b], \mu_{0}\right)}+\left\|g^{(k)}\right\|_{L^{p}\left(\left[a+\delta_{k-1}, b\right], w_{k}\right)},
$$

for all $g \in V^{k, p}([a, b], \mu)$. The proof of this fact is the same as part (1) in Theorem 4.1. We only need to make two remarks:

(a) We need $g^{(k-1)} \in C((a, b])$, and this is true since $w_{k} \in B_{p}((a, b])$.

(b) Lemma 4.1 allows to choose the point $x_{0}$ with $x_{0} \geq a+\delta_{k-1}^{0}$ for some $\delta_{k-1}^{0}$ (independently of $\left.x_{1}, \ldots, x_{k}\right)$. Then it is enough to take $\delta_{k-1}>0$ verifying $0<\delta_{k-1} \leq \delta_{k-1}^{0}$.

We prove now by reverse induction on $j$, with $0 \leq j \leq k-1$, that there exists $\delta_{j}^{0}>0$ such that for each $\delta_{j}$ with $0<\delta_{j} \leq \delta_{j}^{0}$ there exists a positive constant $c_{j}=c_{j}\left(\delta_{j}\right)$ such that

$$
c_{j}\left\|g^{(j)}\right\|_{L^{\infty}\left(\left[a+\delta_{j}, b\right]\right)} \leq\|g\|_{L^{p}\left([a, b], \mu_{0}\right)}+\left\|g^{(k)}\right\|_{L^{p}\left(\left[a+\delta_{j}, b\right], w_{k}\right)},
$$

for all $g \in V^{k, p}([a, b], \mu)$.

Inequality (4.2) gives (4.3) for $j=k-1$. Assume now that (4.3) holds for $j+1$, with $1 \leq j+1 \leq k-1$. Since $1 \in B_{p}((a, b]),(4.2)$ gives, for some $\delta_{j}^{*}>0$, that

$$
\begin{aligned}
d_{1}\left(\delta_{j}\right)\left\|g^{(j)}\right\|_{L^{\infty}\left(\left[a+\delta_{j}, b\right]\right)} & \leq d_{2}\left(\delta_{j}\right)\left(\|g\|_{L^{p}\left([a, b], \mu_{0}\right)}+\left\|g^{(j+1)}\right\|_{L^{p}\left(\left[a+\delta_{j}, b\right]\right)}\right) \\
& \leq\|g\|_{L^{p}\left([a, b], \mu_{0}\right)}+\left\|g^{(j+1)}\right\|_{L^{\infty}\left(\left[a+\delta_{j}, b\right]\right)},
\end{aligned}
$$

for all $g \in V^{k, p}([a, b], \mu)$ and $0<\delta_{j} \leq \delta_{j}^{*}$. This inequality and the induction hypothesis give (4.3) for all $g \in V^{k, p}([a, b], \mu)$ and $0<\delta_{j} \leq \delta_{j+1}^{0}$. Therefore we have the result by defining $\delta_{j}^{0}:=\min \left\{\delta_{j}^{*}, \delta_{j+1}^{0}\right\}$. 
Assume that $a$ is right $j$-regular for some $0 \leq j<k$. Then there exists $\varepsilon>0$ and a right completion $\bar{w}$ of $w$ such that $\bar{w}_{j+1} \in B_{p}([a, a+\varepsilon]$ ) (see Remark 3 to Definition 6). Lemma 3.1 gives

$$
\left\|g^{(j)}(x)-g^{(j)}(a+\varepsilon)\right\|_{L^{\infty}([a, a+\varepsilon])} \leq\left\|g^{(j+1)}\right\|_{L^{1}([a, a+\varepsilon])} \leq c\left\|g^{(j+1)}\right\|_{L^{p}\left([a, a+\varepsilon], \bar{w}_{j+1}\right)} \cdot
$$

This inequality and Lemma 3.3 give

$$
c\left\|g^{(j)}\right\|_{L^{\infty}([a, a+\varepsilon])} \leq\|g\|_{W^{k, p}([a, a+\varepsilon], w)}+\sum_{i=0}^{k-1}\left|g^{(i)}(a+\varepsilon)\right| .
$$

Inequality (4.3) gives for $0 \leq i \leq k-1$

$$
c_{i}\left|g^{(i)}(a+\varepsilon)\right| \leq\|g\|_{L^{p}\left([a, b], \mu_{0}\right)}+\left\|g^{(k)}\right\|_{L^{p}\left([a, b], w_{k}\right)},
$$

and then

$$
c\left\|g^{(j)}\right\|_{L^{\infty}([a, a+\varepsilon])} \leq\|g\|_{W^{k, p}([a, b], \mu)} .
$$

This inequality together with (4.3) give the result.

Corollary 4.1. Let us consider $1 \leq p \leq \infty$ and a p-admissible vectorial measure, $\mu$, in $[a, b]$ such that $(a, b)^{(0)} \cap \operatorname{supp} \mu_{0}$ has at least $k+1$ points and $w_{k} \in B_{p}((a, b))$. If $I_{j}$ is a compact interval contained in $(a, b)^{(j)}$ for $0 \leq j<k$, then

$$
c \sum_{j=0}^{k-1}\left\|f^{(j)}\right\|_{L^{\infty}\left(I_{j}\right)} \leq\|f\|_{W^{k, p}([a, b], \mu)}, \quad \text { for every } f \in V^{k, p}([a, b], \mu) .
$$

Remark. As a consequence of Theorem 4.3 we have that Corollary 4.1 is true even if $(a, b)^{(0)} \cap \operatorname{supp} \mu_{0}$ has only $k$ points, but we cannot use this argument now since Corollary 4.1 is used in the proof of Theorem 4.3.

Proof. Let us consider $k+1$ points $x_{1}<\cdots<x_{k+1}$ in $(a, b)^{(0)} \cap \operatorname{supp} \mu_{0}$. Theorem 4.2 applied to the intervals $\left[a, x_{k}\right]$ and $\left[x_{2}, b\right]$ gives the result. (In fact, we apply to the interval $\left[x_{2}, b\right]$ the symmetric result of Theorem 4.2 for left completions with $w_{k} \in B_{p}\left(\left[x_{2}, b\right)\right)$.)

Corollary 4.2. Let us consider $1 \leq p \leq \infty, w_{k} \in B_{p}((a, b))$ and $w=\left(0, \ldots, 0, w_{k}\right)$. If $I_{j}$ is a compact interval contained in $(a, b)^{(j)}$ for $0 \leq j<k$, then

$$
c \sum_{j=0}^{k-1}\left\|f^{(j)}\right\|_{L^{\infty}\left(I_{j}\right)} \leq\|f\|_{W^{k, p}([a, b], w)}=\left\|f^{(k)}\right\|_{L^{p}\left([a, b], w_{k}\right)},
$$

for every $f \in V^{k, p}([a, b], w)$ with $f(t)=f^{\prime}(t)=\cdots=f^{(k-1)}(t)=0$ for some $t \in(a, b)$.

Proof. Observe that the formula

$$
f(x)=\int_{t}^{x} f^{(k)}(s) \frac{(x-s)^{k-1}}{(k-1) !} d s
$$

together with Lemma 3.1 give for fixed $\varepsilon>0$ with $t \in[a+\varepsilon, b-\varepsilon]$

$$
c\|f\|_{L^{p}([a+\varepsilon, b-\varepsilon])} \leq c\|f\|_{L^{\infty}([a+\varepsilon, b-\varepsilon])} \leq c\left\|f^{(k)}\right\|_{L^{1}([a+\varepsilon, b-\varepsilon])} \leq\left\|f^{(k)}\right\|_{L^{p}\left([a, b], w_{k}\right)} .
$$

Then, for those functions $f$ the norm $\|f\|_{W^{k, p}\left([a, b], w^{*}\right)}$ is comparable to $\|f\|_{W^{k, p}([a, b], w)}$, where the weight $w^{*}=\left(w_{0}^{*}, 0, \ldots, 0, w_{k}\right)$ is defined by $w_{0}^{*}=\chi_{[a+\varepsilon, b-\varepsilon]}$. So, by Corollary 4.1

$$
c \sum_{j=0}^{k-1}\left\|f^{(j)}\right\|_{L^{\infty}\left(I_{j}\right)} \leq c\|f\|_{W^{k, p}\left([a, b], w^{*}\right)} \leq\|f\|_{W^{k, p}([a, b], w)}=\left\|f^{(k)}\right\|_{L^{p}\left([a, b], w_{k}\right)} .
$$

Lemma 4.2. Let us suppose that $1 \leq p \leq \infty$ and $w=\left(w_{0}, \ldots, w_{k}\right)$ is a vectorial weight in $\Omega$. If $I$ is a compact interval contained in $\Omega_{j+1} \cup \cdots \cup \Omega_{k}$ for some $0 \leq j<k$, and $I \cap \Omega_{s} \neq \emptyset$ for some $0 \leq s \leq j$ then there exists a positive constant $c$ such that

$$
c\left\|f^{(j)}\right\|_{L^{\infty}(I)} \leq\left\|f^{(s)}\right\|_{W^{k-s, p}(I, w)} \leq\|f\|_{W^{k, p}(\Omega, w)}, \quad \text { for every } f \in V^{k, p}(\Omega, w) .
$$


Proof. Without loss of generality we can assume that $s=0$ and $I \cap \Omega_{k} \neq \emptyset$.

If $I=[\alpha, \beta]$ then $\alpha, \beta \in \Omega_{j+1} \cup \cdots \cup \Omega_{k}$. Choose now the maximum $\alpha_{1}$ such that $\left[\alpha, \alpha_{1}\right)$ is contained in $\Omega_{i(0)}$ for some $j+1 \leq i(0) \leq k$. If $\alpha_{1} \leq \beta$ we choose the maximum $\alpha_{2}$ such that $\left[\alpha_{1}, \alpha_{2}\right)$ is contained in $\Omega_{i(1)}$ for some $j+1 \leq i(1) \leq k$ and define recursively the intervals $\left[\alpha_{n}, \alpha_{n+1}\right)$ in a similar way if $\alpha_{n} \leq \beta$.

We obtain a finite sequence of numbers $\left\{\alpha_{n}\right\}$, since there exists some $m$ for which $\alpha_{m+1}>\beta$. If this were not true, then we would obtain an infinite increasing sequence $\left\{\alpha_{n}\right\}_{n=1}^{\infty}$ with $\lim _{n \rightarrow \infty} \alpha_{n}=\alpha^{*} \leq \beta$. In that case $\alpha^{*} \in[\alpha, \beta] \subseteq \Omega_{j+1} \cup \cdots \cup \Omega_{k}$ and there exist $\varepsilon>0$ and $j+1 \leq i^{*} \leq k$ with $\left(\alpha^{*}-\varepsilon, \alpha^{*}+\varepsilon\right) \subseteq \Omega_{i^{*}}$. Let us consider $\alpha_{N} \in\left(\alpha^{*}-\varepsilon, \alpha^{*}\right)$; we have $\left[\alpha_{N}, \alpha^{*}+\varepsilon\right) \subseteq \Omega_{i^{*}}$ and then $\alpha_{N+1} \geq \alpha^{*}+\varepsilon>\alpha^{*}$, which contradicts that $\left\{\alpha_{n}\right\}$ is an increasing sequence to $\alpha^{*}$.

As the sets $\Omega_{j+1}, \ldots, \Omega_{k}$ are all open we can choose $\varepsilon>0$ such that $\alpha<\alpha_{1}-2 \varepsilon$ and

$$
\begin{aligned}
H_{0} & :=\left[\alpha, \alpha_{1}-\varepsilon\right] \subseteq \Omega_{i(0)}, \\
H_{1} & :=\left[\alpha_{1}-2 \varepsilon, \alpha_{2}-\varepsilon\right] \subseteq \Omega_{i(1)}, \\
\vdots & \\
H_{m} & :=\left[\alpha_{m}-2 \varepsilon, \beta\right] \subseteq \Omega_{i(m)} .
\end{aligned}
$$

Then $H_{0} \cup \cdots \cup H_{m}=I$ and $\left|H_{r} \cap H_{r+1}\right|=\varepsilon$ for $0 \leq r<m$.

For $j+1 \leq i \leq k$, consider now the sets $L_{r, i}:=H_{r}$ if $H_{r} \subseteq \Omega_{i}$ and $L_{r, i}:=\emptyset$ otherwise, and define also

$$
I_{i}:=\cup_{r=0}^{m} L_{r, i} \subseteq \Omega_{i} .
$$

Obviously $\cup_{i=j+1}^{k} I_{i}=I$ and $w_{i} \in B_{p}\left(I_{i}\right)$ for $j+1 \leq i \leq k$. If we modify slightly our weights by

$$
w^{*}:=\left(w_{0}^{*}, \ldots, w_{k}^{*}\right), \quad w_{k}^{*}:=w_{k}, \quad w_{i}^{*}:=w_{i}+\chi_{I_{i+1} \cup \cdots \cup I_{k}} \quad \text { for } j+1 \leq i<k,
$$

we have that $w_{i}^{*} \in B_{p}\left(I_{i} \cup \cdots \cup I_{k}\right)$. Then we claim

$$
c\left\|f^{(i)}\right\|_{L^{p}\left(I, w_{i}^{*}\right)} \leq\|f\|_{W^{k, p}(I, w)},
$$

for $j+1 \leq i \leq k$ and every $f \in V^{k, p}(\Omega, w)$.

We proceed by reverse induction in $i$, with $j+1 \leq i \leq k$. It is true for $i=k$. Assume now that it is true for $i+1$; we prove now the result for $i$. It is enough to see that

$$
c\left\|f^{(i)}\right\|_{L^{\infty}\left(I_{i+1} \cup \cdots \cup I_{k}\right)} \leq\|f\|_{W^{k, p}(I, w)} .
$$

Observe that the induction hypothesis gives

$$
c\left\|f^{(i+1)}\right\|_{L^{p}\left(I, w_{i+1}^{*}\right)} \leq\|f\|_{W^{k, p}(I, w)} .
$$

Let $M$ be a connected component of $I_{i+1} \cup \cdots \cup I_{k}$. If $M \neq I$, there exists $j+1 \leq l \leq i$ such that $\left|M \cap I_{l}\right| \geq \varepsilon$ since $I$ is connected, and then by Theorem 4.1(2)

$$
c \sum_{h=l}^{i}\left\|f^{(h)}\right\|_{L^{\infty}(M)} \leq\left\|f^{(l)}\right\|_{L^{p}\left(M, w_{l}\right)}+\left\|f^{(i+1)}\right\|_{L^{p}\left(M, w_{i+1}^{*}\right)},
$$

since $w_{i+1}^{*} \in B_{p}\left(I_{i+1} \cup \cdots \cup I_{k}\right)$.

If $M=I$, then $\left|M \cap \Omega_{0}\right|>0$, since $M \cap \Omega_{0}=I \cap \Omega_{0} \neq \emptyset, \Omega_{0}$ is open and $M$ is an interval. Using the previous argument with $l=0$, we obtain

$$
c \sum_{h=0}^{i}\left\|f^{(h)}\right\|_{L^{\infty}(M)} \leq\|f\|_{L^{p}\left(M, w_{0}\right)}+\left\|f^{(i+1)}\right\|_{L^{p}\left(M, w_{i+1}^{*}\right)} .
$$

Consequently, in any case we have

$$
c\left\|f^{(i)}\right\|_{L^{\infty}(M)} \leq\|f\|_{W^{k, p}(M, w)}+\left\|f^{(i+1)}\right\|_{L^{p}\left(M, w_{i+1}^{*}\right)} .
$$

As we only have a finite number of connected components of $I_{i+1} \cup \cdots \cup I_{k}$, the last inequality together with the estimate (4.4) prove the desired result for $i$. 
If we take now $i=j+1$ in the claim we have proved, we obtain

$$
c\left\|f^{(j+1)}\right\|_{L^{p}\left(I, w_{j+1}^{*}\right)} \leq\|f\|_{W^{k, p}(I, w)},
$$

where $w_{j+1}^{*} \in B_{p}\left(I_{j+1} \cup \cdots \cup I_{k}\right)=B_{p}(I)$. Remember that by hypothesis $\left|I \cap \Omega_{0}\right|>0$, and therefore Theorem 4.1(1) gives

$$
c\left\|f^{(j)}\right\|_{L^{\infty}(I)} \leq c\left(\|f\|_{L^{p}\left(I, w_{0}\right)}+\left\|f^{(j+1)}\right\|_{L^{p}\left(I, w_{j+1}^{*}\right)}\right) \leq\|f\|_{W^{k, p}(I, w)} .
$$

This ends our proof.

Lemma 4.3. Let us suppose that $1 \leq p \leq \infty$ and $w=\left(w_{0}, \ldots, w_{k}\right)$ is a vectorial weight in $\Omega$. If $I=[\alpha, \beta]$ is contained in $\Omega^{(j)}$ for some $0 \leq j<k, I \cap \Omega_{s} \neq \emptyset$ for some $0 \leq s \leq j$ and $(\alpha, \beta) \subseteq \Omega_{j+1} \cup \cdots \cup \Omega_{k}$, then there exists a positive constant $c$ such that

$$
c\left\|f^{(j)}\right\|_{L^{\infty}(I)} \leq\left\|f^{(s)}\right\|_{W^{k-s, p}(I, w)} \leq\|f\|_{W^{k, p}(\bar{\Omega}, w)}, \quad \text { for every } f \in V^{k, p}(\bar{\Omega}, w) .
$$

Proof. Without loss of generality we can assume that $s=0$. The case $I \subseteq \Omega_{j+1} \cup \cdots \cup \Omega_{k}$ is the previous lemma. Assume that $\alpha \notin \Omega_{j+1} \cup \cdots \cup \Omega_{k}, \beta \in \Omega_{j+1} \cup \cdots \cup \Omega_{k}$. Since $\alpha$ is right $j$-regular, then there exist $0<\varepsilon<\beta-\alpha$ and a right completion $\bar{w}$ of $w$ such that $\bar{w}_{j+1} \in B_{p}([\alpha, \alpha+\varepsilon])$. By Remark 4 to Definition 6 , if we define $k_{0}:=\max \left\{0 \leq i \leq k: \exists \eta>0\right.$ with $\left.w_{i} \in B_{p}((\alpha, \alpha+\eta])\right\}$, we can choose $\bar{w}$ with $\bar{w}_{i}=w_{i}$ for $k_{0} \leq i \leq k$ and $w_{k_{0}} \in B_{p}((\alpha, \alpha+\varepsilon])$. Obviously we have $j+1 \leq k_{0}$.

Now, Lemma 3.3 gives that

$$
c\left\|f^{(j+1)}\right\|_{L^{p}\left([\alpha, \alpha+\varepsilon], \bar{w}_{j+1}\right)} \leq\|f\|_{W^{k, p}([\alpha, \alpha+\varepsilon], w)}+\sum_{i=j+1}^{k_{0}-1}\left|f^{(i)}(\alpha+\varepsilon)\right|,
$$

if $j+1<k_{0}$. If $j+1=k_{0}$, the sum does not appear and the inequality is trivial since $\bar{w}_{k_{0}}=w_{k_{0}}$. Therefore Lemma 3.1 implies

$$
\begin{aligned}
c\left\|f^{(j)}\right\|_{L^{\infty}([\alpha, \alpha+\varepsilon])} & \leq c\left(\left\|f^{(j+1)}\right\|_{L^{p}\left([\alpha, \alpha+\varepsilon], \bar{w}_{j+1}\right)}+\left|f^{(j)}(\alpha+\varepsilon)\right|\right) \\
& \leq\|f\|_{W^{k, p}([\alpha, \alpha+\varepsilon], w)}+\sum_{i=j}^{k_{0}-1}\left|f^{(i)}(\alpha+\varepsilon)\right|,
\end{aligned}
$$

since $\bar{w}_{j+1} \in B_{p}([\alpha, \alpha+\varepsilon])$. We know there is some $0<\delta<\varepsilon$ such that $J:=[\alpha+\delta, \beta]$ has non-empty intersection with $\Omega_{0}$, so applying Lemma 4.2

$$
c\left\|f^{(j)}\right\|_{L^{\infty}(J)} \leq\|f\|_{W^{k, p}(J, w)},
$$

since $J \subseteq \Omega_{j+1} \cup \cdots \cup \Omega_{k}$. Then Theorem 4.1(2) implies

$$
c \sum_{i=j}^{k_{0}-1}\left|f^{(i)}(\alpha+\varepsilon)\right| \leq c \sum_{i=j}^{k_{0}-1}\left\|f^{(i)}\right\|_{L^{\infty}([\alpha+\delta, \alpha+\varepsilon])} \leq\left\|f^{(j)}\right\|_{L^{p}([\alpha+\delta, \alpha+\varepsilon])}+\left\|f^{\left(k_{0}\right)}\right\|_{L^{p}\left([\alpha+\delta, \alpha+\varepsilon], w_{k_{0}}\right)},
$$

since $w_{k_{0}} \in B_{p}((\alpha, \alpha+\varepsilon]) \subseteq B_{p}([\alpha+\delta, \alpha+\varepsilon])$. Now, applying (4.6) we obtain

$$
c\left\|f^{(j)}\right\|_{L^{p}([\alpha+\delta, \alpha+\varepsilon])} \leq c\left\|f^{(j)}\right\|_{L^{\infty}(J)} \leq\|f\|_{W^{k, p}(J, w)},
$$

and then

$$
c \sum_{i=j}^{k_{0}-1}\left|f^{(i)}(\alpha+\varepsilon)\right| \leq\|f\|_{W^{k, p}(J, w)}
$$

Therefore (4.5) gives

$$
c\left\|f^{(j)}\right\|_{L^{\infty}([\alpha, \alpha+\varepsilon])} \leq\|f\|_{W^{k, p}(I, w)} .
$$

This inequality and (4.6) yield the result. similar.

The cases $\alpha \in \Omega_{j+1} \cup \cdots \cup \Omega_{k}, \beta \notin \Omega_{j+1} \cup \cdots \cup \Omega_{k}$ and $\alpha \notin \Omega_{j+1} \cup \cdots \cup \Omega_{k}, \beta \notin \Omega_{j+1} \cup \cdots \cup \Omega_{k}$ are 
Lemma 4.4. Let us suppose that $1 \leq p \leq \infty$, and that $\mu$ is a $p$-admissible vectorial measure in $\bar{\Omega}$. Assume that $\Omega_{1} \cup \cdots \cup \Omega_{k}$ is connected. Let $K_{j}$ be a finite union of compact intervals contained in $\Omega^{(j)}$, for $0 \leq j<k$. Then there exists an integer $0 \leq l \leq k$, independent of $K_{1}, \ldots, K_{k-1}$, with the following properties:

(1) If $l<k$, there exists a positive constant $c_{0}=c_{0}\left(K_{0}, \ldots, K_{k-1}\right)$ such that

$$
c_{0} \sum_{j=l}^{k-1}\left\|g^{(j)}\right\|_{L^{\infty}\left(K_{j}\right)} \leq\|g\|_{W^{k, p}(\bar{\Omega}, \mu)}, \quad \forall g \in V^{k, p}(\bar{\Omega}, \mu) .
$$

(2) If $l>0, m_{j}:=\#\left\{\operatorname{supp} \mu_{j} \cap \Omega^{(j)}\right\}<\infty$ for $0 \leq j<l$.

Remark. In fact, we can obtain in (2) $m_{j}<l-j$, but the inequality $m_{j}<\infty$ is good enough for our purposes.

Proof. Let us define

$$
A:=\left\{1 \leq j \leq k: \Omega_{j} \neq \emptyset\right\} .
$$

If $A=\emptyset$, we can take $l=0$ and there is nothing to prove, since $K_{j} \subseteq \Omega^{(j)}=\emptyset$. If $A \neq \emptyset$ we define $k_{0}=\min A$. Without loss of generality we can suppose that $\Omega_{k} \neq \emptyset$.

For the case $k_{0}<k$ we claim that

$$
c \sum_{j=k_{0}}^{k-1}\left\|g^{(j)}\right\|_{L^{\infty}\left(K_{j}\right)} \leq\|g\|_{W^{k, p}(\bar{\Omega}, \mu)} .
$$

Let us fix $k_{0} \leq j<k$. In order to prove this claim, without loss of generality we can assume that $K_{j}$ is a single compact interval. The remark before Definition 7 gives that $K_{j} \backslash\left(\Omega_{j+1} \cup \cdots \cup \Omega_{k}\right)$ is a discrete set; since $K_{j}$ is compact, we have that $K_{j} \backslash\left(\Omega_{j+1} \cup \cdots \cup \Omega_{k}\right)$ is finite. Then, we can assume also that $\operatorname{int}\left(K_{j}\right) \subseteq \Omega_{j+1} \cup \cdots \cup \Omega_{k}$.

We can choose a compact interval $I$ with $K_{j} \subseteq I \subseteq \Omega^{(j)}$, int $(I) \subseteq \Omega_{j+1} \cup \cdots \cup \Omega_{k}$ and $I \cap\left(\Omega_{k_{0}} \cup \cdots \cup \Omega_{j}\right) \neq$ $\emptyset$, since $\left(\Omega_{k_{0}} \cup \cdots \cup \Omega_{j}\right) \cup\left(\Omega_{j+1} \cup \cdots \cup \Omega_{k}\right)=\Omega_{1} \cup \cdots \cup \Omega_{k}$ is connected.

Then, there exists a $k_{0} \leq s \leq j$ with $I \cap \Omega_{s} \neq \emptyset$. Lemma 4.3 gives

$$
c\left\|g^{(j)}\right\|_{L^{\infty}(I)} \leq\left\|g^{(s)}\right\|_{W^{k-s, p}(I, w)} \leq\|g\|_{W^{k, p}(\bar{\Omega}, \mu)} .
$$

Consequently, we have (4.7) for $k_{0}<k$.

Define $B:=\left\{0 \leq j<k_{0}: m_{j}=\infty\right\}$ and the non-negative integer $l$ as follows, $l:=\min B$ if $B \neq \emptyset$, and $l:=k_{0}$ if $B=\emptyset$.

If $l=k_{0}$, we have $B=\emptyset$. This implies (2) since $l=k_{0} \geq 1$. If $l=k_{0}<k$, inequality (4.7) gives (1).

We denote by $(\alpha, \beta)$ the interval $\Omega_{1} \cup \cdots \cup \Omega_{k}$.

If $0<l<k_{0}$, we have (2). If $l<k_{0}$, we also have $l<k$ and $m_{l}=\infty$. For each $l \leq j<k_{0}$, we can choose a compact interval $I_{j}$ verifying:

(i) $K_{j} \subseteq I_{j} \subseteq \Omega^{(j)}$

(ii) The minimum point of $I_{j}$ is $\alpha$ if $\alpha$ is right $j$-regular and is $\alpha+\delta$ (with $0<\delta<(\beta-\alpha) / 2$ independent of $j$ ) otherwise.

(iii) The maximum point of $I_{j}$ is $\beta$ if $\beta$ is left $j$-regular and is $\beta-\delta$ otherwise.

(iv) \#\{supp $\left.\mu_{l} \cap[\alpha+\delta, \beta-\delta]\right\} \geq k_{0}-l$.

(Recall that $\Omega^{(l)}, \ldots, \Omega^{\left(k_{0}-1\right)}$ are intervals with the same interior, since $\Omega_{k_{0}} \cup \cdots \cup \Omega_{k}=\Omega_{1} \cup \cdots \cup \Omega_{k}$ is connected.)

We can now finish the proof of Lemma 4.4 in the case $k_{0}<k$. The case $k_{0}=k$ is simpler. 
The remark to Theorem 4.1(2) gives

$$
c \sum_{j=l}^{k_{0}-1}\left\|g^{(j)}\right\|_{L^{\infty}([\alpha+\delta, \beta-\delta])} \leq\left\|g^{(l)}\right\|_{L^{p}\left([\alpha+\delta, \beta-\delta], \mu_{l}\right)}+\left\|g^{\left(k_{0}\right)}\right\|_{L^{1}([\alpha+\delta, \beta-\delta])} .
$$

By the argument at the beginning of the proof of Lemma 4.2 we can split $[\alpha+\delta, \beta-\delta]=E \cup F$, where $E, F$ are finite unions of compact intervals with $E \subseteq \Omega_{k_{0}}$ and $F \subseteq \Omega_{k_{0}+1} \cup \cdots \cup \Omega_{k} \subseteq \Omega^{\left(k_{0}\right)}$. Then Lemma 3.1 gives

$$
c\left\|g^{\left(k_{0}\right)}\right\|_{L^{1}(E)} \leq\left\|g^{\left(k_{0}\right)}\right\|_{L^{p}\left(E, w_{k_{0}}\right)} \leq\|g\|_{W^{k, p}(\bar{\Omega}, \mu)},
$$

since $w_{k_{0}} \in B_{p}(E)$. Inequality (4.7) with $F$ instead of $K_{k_{0}}$ implies

$$
c\left\|g^{\left(k_{0}\right)}\right\|_{L^{1}(F)} \leq c\left\|g^{\left(k_{0}\right)}\right\|_{L^{\infty}(F)} \leq\|g\|_{W^{k, p}(\bar{\Omega}, \mu)},
$$

since $F \subseteq \Omega^{\left(k_{0}\right)}$. So, joining these two inequalities

$$
c\left\|g^{\left(k_{0}\right)}\right\|_{L^{1}([\alpha+\delta, \beta-\delta])} \leq c\left(\left\|g^{\left(k_{0}\right)}\right\|_{L^{1}(E)}+\left\|g^{\left(k_{0}\right)}\right\|_{L^{1}(F)}\right) \leq\|g\|_{W^{k, p}(\bar{\Omega}, \mu)} .
$$

Therefore we conclude

$$
c \sum_{j=l}^{k_{0}-1}\left\|g^{(j)}\right\|_{L^{\infty}([\alpha+\delta, \beta-\delta])} \leq\|g\|_{W^{k, p}(\bar{\Omega}, \mu)} .
$$

We bound now the norm of $g^{(j)}$ in $L^{\infty}([\alpha, \alpha+\delta])$ if $\alpha$ is right $j$-regular. The case $L^{\infty}([\beta-\delta, \beta])$ is symmetric. If $\alpha$ is not right $l$-regular there is nothing to prove. Assume that $\alpha$ is right $j$-regular for some $l \leq j<k$. If we define

$$
k_{1}:=\max \left\{0 \leq i \leq k: \exists \eta>0 \text { with } w_{i} \in B_{p}((\alpha, \alpha+\eta])\right\}
$$

by Remark 4 to Definition 6 there exist $\varepsilon>0$ and a right completion $\bar{w}$ with $\bar{w}_{j+1} \in B_{p}([\alpha, \alpha+\varepsilon])$, $\bar{w}_{k_{1}} \in B_{p}((\alpha, \alpha+\varepsilon])$ and $\bar{w}_{i}=w_{i}$ for $k_{1} \leq i \leq k$. Obviously $k_{1} \geq j+1$.

Lemma 3.3 gives

$$
c\left\|g^{(j+1)}\right\|_{L^{p}\left([\alpha, \alpha+\varepsilon], \bar{w}_{j+1}\right)} \leq\|g\|_{W^{k, p}([\alpha, \alpha+\varepsilon], \mu)}+\sum_{i=j+1}^{k_{1}-1}\left|g^{(i)}(\alpha+\varepsilon)\right| .
$$

Now we conclude with Lemma 3.1

$$
c\left\|g^{(j)}\right\|_{L^{\infty}([\alpha, \alpha+\varepsilon])} \leq\|g\|_{W^{k, p}([\alpha, \alpha+\varepsilon], \mu)}+\sum_{i=j}^{k_{1}-1}\left|g^{(i)}(\alpha+\varepsilon)\right| .
$$

We can assume, perhaps with a smaller $\delta$, that $\alpha+\varepsilon \in[\alpha+\delta, \beta-\delta]$ (this new $\delta$ obviously satisfies properties (i)-(iv)). Inequality (4.8) gives

$$
c \sum_{i=j}^{k_{0}-1}\left|g^{(i)}(\alpha+\varepsilon)\right| \leq\|g\|_{W^{k, p}(\bar{\Omega}, \mu)} .
$$

If $k_{1}>k_{0}$, inequality $(4.7)$ implies

$$
c \sum_{i=k_{0}}^{k_{1}-1}\left|g^{(i)}(\alpha+\varepsilon)\right| \leq\|g\|_{W^{k, p}(\bar{\Omega}, \mu)},
$$

since $\alpha+\varepsilon$ is left $\left(k_{1}-1\right)$-regular. These two last inequalities give

$$
c \sum_{i=j}^{k_{1}-1}\left|g^{(i)}(\alpha+\varepsilon)\right| \leq\|g\|_{W^{k, p}(\bar{\Omega}, \mu)} .
$$

This fact and (4.9) imply

$$
c\left\|g^{(j)}\right\|_{L^{\infty}([\alpha, \alpha+\varepsilon])} \leq\|g\|_{W^{k, p}(\bar{\Omega}, \mu)} .
$$

This finishes the proof of Lemma 4.4. 
Let us define a subspace, $\mathcal{K}(\bar{\Omega}, \mu)$, of $V^{k, p}\left(\overline{\Omega^{(0)}},\left.\mu\right|_{\Omega^{(0)}}\right)$ which plays an important role in the theory.

Definition 10. We define $\mathcal{K}(\bar{\Omega}, \mu)$ as

$$
\mathcal{K}(\bar{\Omega}, \mu):=\left\{g: \Omega^{(0)} \longrightarrow \mathbf{C} / g \in V^{k, p}\left(\overline{\Omega^{(0)}},\left.\mu\right|_{\Omega^{(0)}}\right),\|g\|_{W^{k, p}\left(\overline{\Omega^{(0)}}, \mu_{\Omega^{(0)}}\right.}=0\right\} .
$$

The case in which $\|\cdot\|_{W^{k, p}(\Omega, \mu)}$ is a norm is the most interesting. However we need something more in order to prove part (a) of Theorem 4.3 below: this additional condition is what we present in the following definition of class $\mathcal{C}_{0}$. Roughly speaking, $\mu \in \mathcal{C}_{0}$ if $\|\cdot\|_{W^{k, p}\left(M_{n}, \mu\right)}$ is a norm for some sequence of compact sets $\left\{M_{n}\right\}$ growing to $\Omega$. This condition is exactly what we need since in the proof of Theorem 4.1 we approximate $\Omega$ by compact sets.

If $\mu \notin \mathcal{C}_{0}$ we still can prove part (b) of Theorem 4.3 by adding some Dirac deltas to $\mu_{0}$; we only add the exact amount that we need. This leads to the definition of class $\mathcal{C}$.

Definition 11. Let us consider $1 \leq p \leq \infty$, an open set $\Omega \subseteq \mathbf{R}$ and a $p$-admissible vectorial measure $\mu$ in $\bar{\Omega}$. We say that $(\bar{\Omega}, \mu)$ belongs to the class $\mathcal{C}_{0}$ if there exist compact sets $M_{n}$, which are finite unions of compact intervals, such that

i) $M_{n}$ intersects at most a finite number of connected components of $\Omega_{1} \cup \cdots \cup \Omega_{k}$,

ii) $\mathcal{K}\left(M_{n}, \mu\right)=\{0\}$,

iii) $M_{n} \subseteq M_{n+1}$,

iv) $\cup_{n} M_{n}=\Omega^{(0)}$.

We say that $(\bar{\Omega}, \mu)$ belongs to the class $\mathcal{C}$ if there exists a measure $\mu_{0}^{\prime}=\mu_{0}+\sum_{m \in D} c_{m} \delta_{x_{m}}$ with $c_{m}>0$, $\left\{x_{m}\right\} \subset \Omega^{(0)}, D \subseteq \mathbf{N}$ and $\left(\bar{\Omega}, \mu^{\prime}\right) \in \mathcal{C}_{0}$, where $\mu^{\prime}=\left(\mu_{0}^{\prime}, \mu_{1}, \ldots, \mu_{k}\right)$ is minimal in the following sense: there exists $\left\{M_{n}\right\}$ corresponding to $\left(\bar{\Omega}, \mu^{\prime}\right) \in \mathcal{C}_{0}$ such that if $\mu_{0}^{\prime \prime}=\mu_{0}^{\prime}-c_{m_{0}} \delta_{x_{m_{0}}}$ with $m_{0} \in D$ and $\mu^{\prime \prime}=$ $\left(\mu_{0}^{\prime \prime}, \mu_{1}, \ldots, \mu_{k}\right)$, then $\mathcal{K}\left(M_{n}, \mu^{\prime \prime}\right) \neq\{0\}$ if $x_{m_{0}} \in M_{n}$.

Remarks.

1. The condition on $(\bar{\Omega}, \mu)$ is very general. In fact, the Remark after the proof of Theorem 4.3 below gives that if $\Omega^{(0)} \backslash\left(\Omega_{1} \cup \cdots \cup \Omega_{k}\right)$ has only a finite number of points in each connected component of $\Omega^{(0)}$, then $(\bar{\Omega}, \mu) \in \mathcal{C}$. If, furthermore, $\mathcal{K}(\bar{\Omega}, \mu)=\{0\}$, we have $(\bar{\Omega}, \mu) \in \mathcal{C}_{0}$.

2. Since the restriction of a function of $\mathcal{K}(\bar{\Omega}, \mu)$ to $M_{n}$ is in $\mathcal{K}\left(M_{n}, \mu\right)$ for every $n$, then $(\bar{\Omega}, \mu) \in \mathcal{C}_{0}$ implies $\mathcal{K}(\bar{\Omega}, \mu)=\{0\}$.

3. If $(\bar{\Omega}, \mu) \in \mathcal{C}_{0}$, then $(\bar{\Omega}, \mu) \in \mathcal{C}$, with $\mu^{\prime}=\mu$.

4. The proof of Theorem 4.3 below gives that if for every connected component $\Lambda$ of $\Omega_{1} \cup \cdots \cup \Omega_{k}$ we have $\mathcal{K}(\bar{\Lambda}, \mu)=\{0\}$, then $(\bar{\Omega}, \mu) \in \mathcal{C}_{0}$. Condition \#supp $\left.\mu_{0}\right|_{\bar{\Lambda} \cap \Omega^{(0)}} \geq k$ implies $\mathcal{K}(\bar{\Lambda}, \mu)=\{0\}$.

The following result is the main ingredient of the proof of Theorem 5.1, which is the most important result that we present in this paper. It is also useful by its applications in the papers [RARP], [R1], [R2], [R3] and [APRR]. Furthermore, it is important by itself, since it answers to the following main question: when the evaluation functional of $f\left(\right.$ or $\left.f^{(j)}\right)$ in a point is a bounded operator in $W^{k, p}(\bar{\Omega}, \mu)$ ?

Theorem 4.3. Let us consider $1 \leq p \leq \infty$, an open set $\Omega \subseteq \mathbf{R}$ and a $p$-admissible vectorial measure $\mu$ in $\bar{\Omega}$. Let $K_{j}$ be a finite union of compact intervals contained in $\Omega^{(j)}$, for $0 \leq j<k$ and $\bar{w}$ a right (or left) completion of $w$. Then:

(a) If $(\bar{\Omega}, \mu) \in \mathcal{C}_{0}$ there exist positive constants $c_{1}=c_{1}\left(K_{0}, \ldots, K_{k-1}\right)$ and $c_{2}=c_{2}\left(\bar{w}, K_{0}, \ldots, K_{k-1}\right)$ such that

$$
c_{1} \sum_{j=0}^{k-1}\left\|g^{(j)}\right\|_{L^{\infty}\left(K_{j}\right)} \leq\|g\|_{W^{k, p}(\bar{\Omega}, \mu)}, \quad c_{2}\|g\|_{W^{k, p}(\bar{\Omega}, \bar{w})} \leq\|g\|_{W^{k, p}(\bar{\Omega}, \mu)}, \quad \forall g \in V^{k, p}(\bar{\Omega}, \mu) .
$$

(b) If $(\bar{\Omega}, \mu) \in \mathcal{C}$ there exist positive constants $c_{3}=c_{3}\left(K_{0}, \ldots, K_{k-1}\right)$ and $c_{4}=c_{4}\left(\bar{w}, K_{0}, \ldots, K_{k-1}\right)$ such that for every $g \in V^{k, p}(\bar{\Omega}, \mu)$, there exists $g_{0} \in V^{k, p}(\bar{\Omega}, \mu)$, independent of $K_{0}, \ldots, K_{k-1}, c_{3}, c_{4}$ and $\bar{w}$, with

$$
\left\|g_{0}-g\right\|_{W^{k, p}(\bar{\Omega}, \mu)}=0,
$$




$$
c_{3} \sum_{j=0}^{k-1}\left\|g_{0}^{(j)}\right\|_{L^{\infty}\left(K_{j}\right)} \leq\left\|g_{0}\right\|_{W^{k, p}(\bar{\Omega}, \mu)}=\|g\|_{W^{k, p}(\bar{\Omega}, \mu)}, \quad c_{4}\left\|g_{0}\right\|_{W^{k, p}(\bar{\Omega}, \bar{w})} \leq\|g\|_{W^{k, p}(\bar{\Omega}, \mu)} .
$$

Furthermore, if $g_{0}, f_{0}$ are these representatives of $g, f$ respectively, we have for the same constants $c_{3}, c_{4}$

$$
c_{3} \sum_{j=0}^{k-1}\left\|g_{0}^{(j)}-f_{0}^{(j)}\right\|_{L^{\infty}\left(K_{j}\right)} \leq\|g-f\|_{W^{k, p}(\bar{\Omega}, \mu)}, \quad c_{4}\left\|g_{0}-f_{0}\right\|_{W^{k, p}(\bar{\Omega}, \bar{w})} \leq\|g-f\|_{W^{k, p}(\bar{\Omega}, \mu)} .
$$

Proof of Theorem 4.3. Without loss of generality we can assume that $\Omega^{(0)}$ is connected. This condition is not restrictive since otherwise we can work in each connected component. Let us consider the connected components $\left\{\Lambda_{\lambda}\right\}_{\lambda \in A}$ of $\Omega_{1} \cup \cdots \cup \Omega_{k}$. Recall that $\Omega^{(0)} \backslash\left(\Omega_{1} \cup \cdots \cup \Omega_{k}\right)$ is a discrete set since $\Omega^{(0)}$ is connected (see the Remark before Definition 7 ). Moreover this set cannot have any accumulation point in $\Omega^{(0)}$. Therefore we can take the set of indices $A$ as one of the following sets: $\mathbf{Z}, \mathbf{Z}^{+}, \mathbf{Z}^{-}$or $\{1, \ldots, N\}$ for some natural number $N$, with the property that $\sup \Lambda_{\lambda}=\inf \Lambda_{\lambda+1}$ if $\lambda, \lambda+1 \in A$.

For $0 \leq j<k$ consider the compact set $K_{j} \subseteq \Omega^{(j)}$, which is a finite union of intervals. It contains at most a finite number of points which are not in $\Omega_{1} \cup \cdots \cup \Omega_{k}$, since $\Omega^{(0)} \backslash\left(\Omega_{1} \cup \cdots \cup \Omega_{k}\right)$ is a discrete set and $K_{j}$ is compact. Therefore each $K_{j}$ is contained at most in the union of a finite number of $\bar{\Lambda}_{\lambda}$.

Let us fix $\lambda \in A$. From now on, we work on $\Lambda_{\lambda}$ (which may have infinite length). Denote by $J_{j}$ the finite union of compact intervals $J_{j}:=\overline{K_{j} \cap \Lambda_{\lambda}}$. In each $\bar{\Lambda}_{\lambda}$, Lemma 4.4 gives that there exist $c, l$ (depending on $\lambda$ ) such that

$$
c \sum_{j=l}^{k-1}\left\|g^{(j)}\right\|_{L^{\infty}\left(J_{j}\right)} \leq\|g\|_{W^{k, p}\left(\bar{\Lambda}_{\lambda}, \mu\right)},
$$

if $l<k$. If $l>0$ we have the additional condition $m_{j}:=\#\left\{\operatorname{supp} \mu_{j} \cap \Lambda_{\lambda}^{(j)}\right\}<\infty$ for $0 \leq j<l$, and so in $\Lambda_{\lambda}^{(j)}$ we can write each $\mu_{j}$ as

$$
\begin{array}{ccc}
\mu_{0} & =a_{0}^{1} \delta_{x_{0}^{1}}+\cdots+a_{0}^{m_{0}} \delta_{x_{0}^{m_{0}}}, \\
\mu_{1} & =a_{1}^{1} \delta_{x_{1}^{1}}+\cdots+a_{1}^{m_{1}} \delta_{x_{1}^{m_{1}}}, \\
\vdots & \vdots & \vdots \\
\mu_{l-1} & =a_{l-1}^{1} \delta_{x_{l-1}^{1}}+\cdots+a_{l-1}^{m_{l-1}} \delta_{x_{l-1}^{m_{l-1}}},
\end{array}
$$

with $a_{j}^{i}>0$ and $x_{j}^{i} \in \Lambda_{\lambda}^{(j)}$ for $0 \leq j<l, 1 \leq i \leq m_{j}$. Without loss of generality we can assume that $J_{j}$ is an interval and $x_{j}^{i} \in J_{j}$ for $0 \leq j<l, 1 \leq i \leq m_{j}$ (as in the proof of Lemma 4.4). If $\Lambda_{\lambda}=(\alpha, \beta)$, we can assume also that $\alpha \in J_{j}$ if $\alpha$ is right $j$-regular and $\beta \in J_{j}$ if $\beta$ is left $j$-regular.

We now study the structure of $\mathcal{K}(\bar{\Omega}, \mu)$. Let $q$ be a function in $\mathcal{K}(\bar{\Omega}, \mu)$. Let us prove that $q^{(k)} \equiv 0$ in each $\Lambda_{\lambda}$ :

If for some $\lambda$ we have $l=k$, then $\Lambda_{\lambda} \subseteq \Omega_{k}$. Since $q \in \mathcal{K}(\bar{\Omega}, \mu)$ we have that

$$
\int_{\Lambda_{\lambda}}\left|q^{(k)}(x)\right|^{p} w_{k}(x) d x=0
$$

Lemma 3.1 gives that $\left\|q^{(k)}\right\|_{L^{1}(I)}=0$, for every compact interval $I \subseteq \Lambda_{\lambda}$; then $\left\|q^{(k)}\right\|_{L^{1}\left(\Lambda_{\lambda}\right)}=0$ and since $q^{(k-1)}$ is locally absolutely continuous in $\Lambda_{\lambda}$, it has to be constant there, and consequently $q^{(k)} \equiv 0$ in $\Lambda_{\lambda}$. Then $\left.q\right|_{\Lambda_{\lambda}} \in P_{k-1}$, since $\Lambda_{\lambda}$ is a connected set.

If for some $\lambda$ we have $l<k$, Lemma 4.4 gives $c\left\|q^{(l)}\right\|_{L^{\infty}(J)} \leq\|q\|_{W^{k, p}\left(\bar{\Lambda}_{\lambda}, \mu\right)}=0$ for any compact interval $J \subseteq \Lambda_{\lambda}^{(l)}$. Then $q^{(l)} \equiv 0$ in any compact interval $J \subseteq \Lambda_{\lambda}^{(l)}$ and we have $q^{(l)} \equiv 0$ in $\Lambda_{\lambda}^{(l)}$ and so, $\left.q\right|_{\Lambda_{\lambda}} \in P_{l-1}$. 
Consequently, in any case $(0 \leq l \leq k)$ we obtain $\left.q\right|_{\Lambda_{\lambda}} \in P_{l-1}$ if we define $P_{-1}:=\{0\}$. Besides, in each connected component $\Lambda_{\lambda}$ one has

$$
q^{(j)}\left(x_{j}^{i}\right)=0 \quad \text { for } 0 \leq j<l, 1 \leq i \leq m_{j} .
$$

If we write in $\Lambda_{\lambda}$

$$
q(x)=\alpha_{l-1} x^{l-1}+\cdots+\alpha_{1} x+\alpha_{0},
$$

we see that (4.10) is a homogeneous linear system of $m_{0}+\cdots+m_{l-1}$ equations with the $l$ unknowns $\alpha_{l-1}, \ldots, \alpha_{1}, \alpha_{0}$, whose solution represents the restriction of the functions in $\mathcal{K}(\bar{\Omega}, \mu)$ to $\Lambda_{\lambda}$ in the basis $\left\{x^{l-1}, \ldots, x, 1\right\}$ of $P_{l-1}$. Observe that the coefficients $\alpha_{i}$ obviously depend on $\lambda$.

On the other hand, if $\lambda, \lambda+1 \in A$ and $\beta=\sup \Lambda_{\lambda}=\inf \Lambda_{\lambda+1}$, we have

$$
q^{(j)}\left(\beta^{-}\right)=q^{(j)}\left(\beta^{+}\right), \quad \text { if } \beta \text { is } j \text {-regular, }
$$

where as usual $q^{(j)}\left(\beta^{-}\right)$and $q^{(j)}\left(\beta^{+}\right)$denotes respectively the left and right derivatives.

Then we have that $\mathcal{K}(\bar{\Omega}, \mu)$ is the solution of the system given by (4.10) for every $\lambda \in A$ and (4.11) for every $\lambda \in A$ such that $\lambda+1 \in A$. Consequently the elements of $\mathcal{K}(\bar{\Omega}, \mu)$ are splines.

Claim. If $\Lambda_{\lambda}=(\alpha, \beta)$, then given any weight $w^{*}$ which is a right completion of $w$ with respect to $\alpha$ and a left completion of $w$ with respect to $\beta$, there exists a positive constant $c$ such that

$$
\left\|g^{(l)}\right\|_{L^{p}\left([\alpha, \beta], w_{l}^{*}\right)} \leq c\|g\|_{W^{k, p}([\alpha, \beta], \mu)}, \quad \text { for every } g \in V^{k, p}([\alpha, \beta], \mu) .
$$

Lemma 3.3 gives if $l<k$

$$
c\left\|g^{(l)}\right\|_{L^{p}\left([\alpha, \alpha+\varepsilon], w_{l}^{*}\right)} \leq\|g\|_{W^{k, p}([\alpha, \alpha+\varepsilon], w)}+\sum_{i=l}^{k-1}\left|g^{(i)}(\alpha+\varepsilon)\right|,
$$

for some $0<\varepsilon<\beta-\alpha$, with $\alpha+\varepsilon \in \Omega_{k}$. If we take $0<\delta<\varepsilon$, with $[\alpha+\delta, \alpha+\varepsilon] \subseteq \Omega_{k} \subseteq \Omega^{(k-1)} \subseteq \cdots \subseteq \Omega^{(l)}$, Lemma 4.4 gives

$$
\sum_{i=l}^{k-1}\left|g^{(i)}(\alpha+\varepsilon)\right| \leq \sum_{i=l}^{k-1}\left\|g^{(i)}\right\|_{L^{\infty}([\alpha+\delta, \alpha+\varepsilon])} \leq c\|g\|_{W^{k, p}([\alpha, \beta], \mu)} .
$$

We conclude

$$
\left\|g^{(l)}\right\|_{L^{p}\left([\alpha, \alpha+\varepsilon], w_{l}^{*}\right)} \leq c\|g\|_{W^{k, p}([\alpha, \beta], \mu)},
$$

for $l \leq k$ (the case $l=k$ is immediate since then $w_{k}^{*}=w_{k}$ ).

In a similar way we have

$$
\left\|g^{(l)}\right\|_{L^{p}\left([\alpha+\varepsilon, \beta], w_{l}^{*}\right)} \leq c\|g\|_{W^{k, p}([\alpha, \beta], \mu)} .
$$

The last inequalities finish the proof of the claim.

In order to prove (a) we can choose $n \in \mathbf{N}$ with $K_{0} \cup \cdots \cup K_{k-1} \subseteq M_{n}$ and $\mathcal{K}\left(M_{n}, \mu\right)=\{0\}$, since $(\bar{\Omega}, \mu) \in \mathcal{C}_{0}$. We denote by $A_{n}$ the finite set of indices $\lambda \in A$ with $\Lambda_{\lambda} \cap M_{n} \neq \emptyset$. Then the finite linear system of equations, which we denote by $T_{n}$, given by (4.10) and (4.11) for $\lambda \in A_{n}$ such that every point appearing in the equations belongs to $M_{n}$, has unique solution.

Given $g \in V^{k, p}(\bar{\Omega}, \mu)$, for each $\lambda \in A_{n}$ fix $a \in M_{n} \cap \operatorname{int}\left(J_{0}\right)$ and let $f$ be the function defined in each $\Lambda_{\lambda}=(\alpha, \beta)$ by $f(x):=g(x)$ if $l=0$, and

$$
f(x):=\int_{a}^{x} g^{(l)}(t) \frac{(x-t)^{l-1}}{(l-1) !} d t,
$$

otherwise. In each $\Lambda_{\lambda}$, we have $g=f+r$ with $r:=0$ if $l=0$, and

$$
r(x):=g(a)+g^{\prime}(a)(x-a)+\cdots+g^{(l-1)}(a) \frac{(x-a)^{l-1}}{(l-1) !},
$$


otherwise. For $0 \leq j<l$ we have

$$
f^{(j)}(x)=\int_{a}^{x} g^{(l)}(t) \frac{(x-t)^{l-j-1}}{(l-j-1) !} d t \quad \text { and } \quad f^{(l)}(x)=g^{(l)}(x)
$$

in $\Lambda_{\lambda}$. Consequently it is clear using Corollary 4.2 , Lemma 4.4 and the claim that there exist positive constants which are independent of $g$ and $f$ such that

$$
\begin{aligned}
\sum_{j=0}^{k-1}\left\|f^{(j)}\right\|_{L^{\infty}\left(J_{j}\right)} & =\sum_{j=0}^{l-1}\left\|f^{(j)}\right\|_{L^{\infty}\left(J_{j}\right)}+\sum_{j=l}^{k-1}\left\|g^{(j)}\right\|_{L^{\infty}\left(J_{j}\right)} \\
& \leq c\left(\left\|g^{(l)}\right\|_{L^{p}\left(\bar{\Lambda}_{\lambda}, w_{l}^{*}\right)}+\|g\|_{W^{k, p}\left(\bar{\Lambda}_{\lambda}, \mu\right)}\right) \\
& \leq c\|g\|_{W^{k, p}\left(\bar{\Lambda}_{\lambda}, \mu\right)}
\end{aligned}
$$

with $w^{*}$ a suitable completion of $w$. Observe that if $l=0$ or $l=k$, the sum $\sum_{j=0}^{l-1}$ or $\sum_{j=l}^{k-1}$ does not appear in (4.12).

If $0 \leq j<l$ and $H:=\min _{i, j, \lambda} a_{j}^{i}$ (for $0 \leq j<l, 1 \leq i \leq m_{j}$ and $\lambda \in A_{n}$ ), we obtain for $x_{j}^{i} \in M_{n}$

$$
\left|g^{(j)}\left(x_{j}^{i}\right)\right|^{p} \leq H^{-1} a_{j}^{i}\left|g^{(j)}\left(x_{j}^{i}\right)\right|^{p} \leq H^{-1}\left\|g^{(j)}\right\|_{L^{p}\left(M_{n}, \mu_{j}\right)}^{p}, \quad \text { for } 1 \leq p<\infty
$$

and

$$
\left|g^{(j)}\left(x_{j}^{i}\right)\right| \leq\left\|g^{(j)}\right\|_{L^{\infty}\left(M_{n}, \mu_{j}\right)}, \quad \text { for } p=\infty .
$$

Therefore, using also (4.12), we have for $1 \leq p \leq \infty$,

$$
\left|r^{(j)}\left(x_{j}^{i}\right)\right|=\left|g^{(j)}\left(x_{j}^{i}\right)-f^{(j)}\left(x_{j}^{i}\right)\right| \leq H^{-1 / p}\left\|g^{(j)}\right\|_{L^{p}\left(M_{n}, \mu_{j}\right)}+c\|g\|_{W^{k, p}\left(R_{n}, \mu\right)},
$$

where $R_{n}:=\cup_{\lambda \in A_{n}} \bar{\Lambda}_{\lambda}$, for $0 \leq j<l, 1 \leq i \leq m_{j}$ and $\lambda \in A_{n}$ with $x_{j}^{i} \in M_{n}$, that is, there exists a positive constant $c$, which is independent of $g$ and $r$ such that

$$
c\left|r^{(j)}\left(x_{j}^{i}\right)\right| \leq\|g\|_{W^{k, p}\left(R_{n}, \mu\right)}, \quad \text { for } 0 \leq j<l, 1 \leq i \leq m_{j}, \lambda \in A_{n}, \text { with } x_{j}^{i} \in M_{n} .
$$

If $\lambda, \lambda+1 \in A_{n}$ and $\beta=\sup \Lambda_{\lambda}=\inf \Lambda_{\lambda+1}$ is $j$-regular, we have

$$
r^{(j)}\left(\beta^{-}\right)=g^{(j)}(\beta)-f^{(j)}\left(\beta^{-}\right), \quad r^{(j)}\left(\beta^{+}\right)=g^{(j)}(\beta)-f^{(j)}\left(\beta^{+}\right),
$$

and then

$$
r^{(j)}\left(\beta^{-}\right)-r^{(j)}\left(\beta^{+}\right)=f^{(j)}\left(\beta^{+}\right)-f^{(j)}\left(\beta^{-}\right) .
$$

Recall that if $\Lambda_{\lambda}=(\alpha, \beta)$, we are assuming that $\alpha \in J_{j}$ if $\alpha$ is right $j$-regular and $\beta \in J_{j}$ if $\beta$ is left $j$-regular. Consequently (4.12) gives

$$
c\left|r^{(j)}\left(\beta^{-}\right)-r^{(j)}\left(\beta^{+}\right)\right| \leq\|g\|_{W^{k, p}\left(R_{n}, \mu\right)} .
$$

The function $\left.r\right|_{M_{n}}$ is determined by a finite linear system whose coefficient matrix is the coefficient matrix of $T_{n}$ and whose non-homogeneous terms are bounded by (4.13) and (4.14), since $T_{n}$ has unique solution.

Consequently, there is a positive constant $c$, independent of $\lambda \in A_{n}, g$ and $r$, such that

$$
c \sum_{j=0}^{k-1}\left\|r^{(j)}\right\|_{L^{\infty}\left(K_{j}\right)} \leq\|g\|_{W^{k, p}\left(R_{n}, \mu\right)} .
$$

Inequalities (4.12) (with $\lambda \in A_{n}$ ) and (4.15) and the relation $g=f+r$ give the first inequality of (a) in Theorem 4.3. The second inequality of (a) is an immediate consequence of the first one and Lemma 3.3. 
We deal now with part (b). Since $(\bar{\Omega}, \mu)$ belongs to the class $\mathcal{C}$, there exist a measure $\mu_{0}^{\prime}=\mu_{0}+$ $\sum_{m \in D} c_{m} \delta_{x_{m}}$ with $c_{m}>0,\left\{x_{m}\right\} \subseteq \Omega^{(0)}$ and $\left(\bar{\Omega}, \mu^{\prime}\right) \in \mathcal{C}_{0}$, where $\mu^{\prime}=\left(\mu_{0}^{\prime}, \mu_{1}, \ldots, \mu_{k}\right)$ is minimal. We choose $\left\{M_{n}\right\}$ corresponding to $\left(\bar{\Omega}, \mu^{\prime}\right) \in \mathcal{C}_{0}$ such that we can apply the minimality of $\mu^{\prime}$.

If $g \in V^{k, p}(\bar{\Omega}, \mu)$ we can choose $r \in \mathcal{K}(\bar{\Omega}, \mu)$ (defined as 0 in the complement of $\Omega^{(0)}$ ) with $\| g-$ $r\left\|_{W^{k, p}\left(\bar{\Omega}, \mu^{\prime}\right)}=\right\| g \|_{W^{k, p}(\bar{\Omega}, \mu)}$, i.e. such that $\|g-r\|_{L^{p}\left(\Omega^{(0)}, \mu_{0}^{\prime}-\mu_{0}\right)}=0$, that is, $g\left(x_{m}\right)=r\left(x_{m}\right)$ for every $m \in D$. To see this we proceed in the following way: as in the proof of part (a), for each $n$ consider the finite linear system of equations $T_{n}$ which describes $q \in \mathcal{K}\left(M_{n}, \mu^{\prime}\right)$ and the system $T_{n}^{*}$ obtained by changing in $T_{n}$ the equations of the form $q\left(x_{m}\right)=0$ with $m \in D$ by $q\left(x_{m}\right)=g\left(x_{m}\right)$. Every solution of $T_{n}^{*}$ belongs to $\mathcal{K}\left(M_{n}, \mu\right)$.

There exists a unique function $r_{n} \in \mathcal{K}\left(M_{n}, \mu\right)$ satisfying $T_{n}^{*}$ : By the minimality of $\mu^{\prime}$, each equation in $T_{n}$ of the form $q\left(x_{m}\right)=0$ with $m \in D$ is linearly independent of the other equations in $T_{n}$. This implies the existence of $r_{n}$. We also have the uniqueness of $r_{n}$ since $T_{n}$ has a unique solution (recall that $\left.\mathcal{K}\left(M_{n}, \mu^{\prime}\right)=\{0\}\right)$.

Observe that every equation of $T_{n}^{*}$ is in $T_{n+1}^{*}$, since $M_{n} \subseteq M_{n+1}$, and therefore $\left.r_{n+1}\right|_{M_{n}}=r_{n}$. Then, we can define $r$ in $\Omega^{(0)}$ by $r(x):=r_{n}(x)$ if $x \in M_{n}$. So $r \in \mathcal{K}(\bar{\Omega}, \mu)$ since $r \in \cap_{n} \mathcal{K}\left(M_{n}, \mu\right)$ and every equation of $\mathcal{K}(\bar{\Omega}, \mu)$ is in some $T_{n}$, and also $r\left(x_{m}\right)=g\left(x_{m}\right)$ for every $m \in D$.

The function $g_{0}:=g-r$ satisfies $\left\|g_{0}-g\right\|_{W^{k, p}(\bar{\Omega}, \mu)}=\|r\|_{W^{k, p}(\bar{\Omega}, \mu)}=0$. We have $K_{j} \subseteq \Omega^{(j)}$ for $0 \leq j<k$ (observe that $\Omega^{(j)}$ is the same for $W^{k, p}(\bar{\Omega}, \mu)$ and $W^{k, p}\left(\bar{\Omega}, \mu^{\prime}\right)$ ). Then we have

$$
\begin{gathered}
c_{3} \sum_{j=0}^{k-1}\left\|g_{0}^{(j)}\right\|_{L^{\infty}\left(K_{j}\right)} \leq\left\|g_{0}\right\|_{W^{k, p}\left(\bar{\Omega}, \mu^{\prime}\right)}=\left\|g_{0}\right\|_{W^{k, p}(\bar{\Omega}, \mu)}=\|g\|_{W^{k, p}(\bar{\Omega}, \mu)}, \\
c_{4}\left\|g_{0}\right\|_{W^{k, p}(\bar{\Omega}, \bar{w})} \leq\left\|g_{0}\right\|_{W^{k, p}\left(\bar{\Omega}, \mu^{\prime}\right)}=\|g\|_{W^{k, p}(\bar{\Omega}, \mu)},
\end{gathered}
$$

since $g_{0}\left(x_{m}\right)=0$ for every $m \in D$.

The other inequalities in (b) can be proved in a similar way.

This finishes the proof of Theorem 4.3.

Remark. In order to prove Remark 1 to Definition 11 we can assume that $\Omega^{(0)}$ is connected. Then, if $\Omega^{(0)} \backslash\left(\Omega_{1} \cup \cdots \cup \Omega_{k}\right)$ has only a finite number of points, we can split $\Omega_{1} \cup \cdots \cup \Omega_{k}=\Lambda_{1} \cup \cdots \cup \Lambda_{N}$ in its connected components. Now, the result is trivial since the linear system that defines $\mathcal{K}(\bar{\Omega}, \mu)$ is finite.

We obtain the following corollary of Theorem 4.3.

Corollary 4.3. Let us consider $1 \leq p \leq \infty$, an open set $\Omega \subseteq \mathbf{R}$ and a p-admissible vectorial measure $\mu$ in $\bar{\Omega}$. Let $K_{j}$ be a finite union of compact intervals contained in $\Omega^{(j)}$, for $0 \leq j<k$. Then:

(a) If $(\bar{\Omega}, \mu) \in \mathcal{C}_{0}$ there exists a positive constant $c_{1}=c_{1}\left(K_{0}, \ldots, K_{k-1}\right)$ such that

$$
c_{1} \sum_{j=0}^{k-1}\left\|g^{(j+1)}\right\|_{L^{1}\left(K_{j}\right)} \leq\|g\|_{W^{k, p}(\bar{\Omega}, \mu)}, \quad \forall g \in V^{k, p}(\bar{\Omega}, \mu) .
$$

(b) If $(\bar{\Omega}, \mu) \in \mathcal{C}$ there exists a positive constant $c_{2}=c_{2}\left(K_{0}, \ldots, K_{k-1}\right)$ such that for every $g \in$ $V^{k, p}(\bar{\Omega}, \mu)$, there exists $g_{0} \in V^{k, p}(\bar{\Omega}, \mu)$ (the same function as in Theorem 4.3), with

$$
\left\|g_{0}-g\right\|_{W^{k, p}(\bar{\Omega}, \mu)}=0, \quad c_{2} \sum_{j=0}^{k-1}\left\|g_{0}^{(j+1)}\right\|_{L^{1}\left(K_{j}\right)} \leq\left\|g_{0}\right\|_{W^{k, p}(\bar{\Omega}, \mu)}=\|g\|_{W^{k, p}(\bar{\Omega}, \mu)} .
$$

Furthermore, if $g_{0}, f_{0}$ are these representatives of $g, f$ respectively, we have for the same constant $c_{2}$

$$
c_{2} \sum_{j=0}^{k-1}\left\|g_{0}^{(j+1)}-f_{0}^{(j+1)}\right\|_{L^{1}\left(K_{j}\right)} \leq\|g-f\|_{W^{k, p}(\bar{\Omega}, \mu)} .
$$


Proof. We only prove part (a) since (b) is similar. Fix $0 \leq j<k$. Since $K_{j} \subseteq \Omega^{(j)}$, given any point $y \in K_{j}$, there exist an interval $J_{y}$ and a completion $\bar{w}^{y}$ of $w$ with $\bar{w}_{j+1}^{y} \in B_{p}\left(J_{y}\right)$. The compactness of $K_{j}$ gives that there exists a finite set of points $y_{1}, \ldots, y_{l}$ with $K_{j} \subseteq J_{y_{1}} \cup \cdots \cup J_{y_{l}}$.

If we define $w_{j+1}^{*}:=\sum_{i=1}^{l} \bar{w}_{j+1}^{y_{i}} \chi_{J_{y_{i}}}$, the second inequality in Theorem 4.3(a) gives

$$
c\left\|g^{(j+1)}\right\|_{L^{p}\left(K_{j}, w_{j+1}^{*}\right)} \leq\|g\|_{W^{k, p}(\bar{\Omega}, \mu)},
$$

and Lemma 3.1 finishes the proof, since $w_{j+1}^{*} \in B_{p}\left(K_{j}\right)$.

We have another corollary.

Corollary 4.4. Let us suppose that $1 \leq p \leq \infty$ and that $\mu=\left(\mu_{0}, \ldots, \mu_{k}\right)$ is a $p$-admissible vectorial measure in $[a, b]$ with $w_{k} \in B_{p}([a, b])$. Then:

(a) There exists a positive constant $c_{1}$ such that

$$
c_{1} \sum_{j=0}^{k-1}\left\|g^{(j)}\right\|_{L^{\infty}([a, b])} \leq\|g\|_{W^{k, p}([a, b], \mu)}, \quad \forall g \in V^{k, p}([a, b], \mu),
$$

if and only if $\mathcal{K}([a, b], \mu)=\{0\}$.

(b) There exists a positive constant $c_{2}$ such that for every $g \in V^{k, p}([a, b], \mu)$, there exists $g_{0}$ with

$$
\left\|g_{0}-g\right\|_{W^{k, p}([a, b], \mu)}=0, \quad c_{2} \sum_{j=0}^{k-1}\left\|g_{0}^{(j)}\right\|_{L^{\infty}([a, b])} \leq\left\|g_{0}\right\|_{W^{k, p}([a, b], \mu)}=\|g\|_{W^{k, p}([a, b], \mu)} .
$$

Proof. Since $\Omega^{(0)}=[a, b]$ and $\Omega^{(0)} \backslash\left(\Omega_{1} \cup \cdots \cup \Omega_{k}\right)=\{a\} \cup\{b\}$ is finite, Remark 1 to Definition 11 says that $([a, b], \mu) \in \mathcal{C}$. Then, if we take $K_{j}=[a, b]$ for $0 \leq j<k$, Theorem 4.3(b) gives (b).

If $\mathcal{K}([a, b], \mu)=\{0\}$, we also have $([a, b], \mu) \in \mathcal{C}_{0}$ by Remark 1 to Definition 11 , with the choice $M_{n}=$ $[a, b]$. Then, Theorem 4.3(a) gives one implication of (a). For the other implication, it is enough to remark that if there exists $g \in V^{k, p}([a, b], \mu)$ not identically zero with $\|g\|_{W^{k, p}([a, b], \mu)}=0$, the inequality $\|g\|_{L^{\infty}[a, b]} \leq$ 0 does not hold.

Corollary 4.4 gives the following result about comparable norms.

Corollary 4.5. Let us consider $1 \leq p<\infty, \mu, \nu$ finite $p$-admissible vectorial measures in $[a, b]$, with absolutely continuous parts $w, v$, respectively. Assume that $w_{k} \in B_{p}([a, b]), w_{k} \asymp v_{k}$ and $\mathcal{K}([a, b], \mu)=$ $\mathcal{K}([a, b], \nu)=\{0\}$. Then:

(1) The norm in $W^{k, p}([a, b], \mu)$ of any function $g \in V^{k, p}([a, b], \mu)$ is comparable to

$$
\sum_{j=0}^{k-1}\left\|g^{(j)}\right\|_{L^{\infty}([a, b])}+\left\|g^{(k)}\right\|_{L^{p}\left([a, b], w_{k}\right)} .
$$

(2) The norms in $W^{k, p}([a, b], \mu)$ and $W^{k, p}([a, b], \nu)$ are comparable.

Remark. The case $p=\infty$ is also true if we change the hypothesis $\mu, \nu$ finite by $w_{0}, \ldots, w_{k-1}, v_{0}, \ldots, v_{k-1} \in$ $L^{\infty}([a, b])$.

\section{Main result.}

Theorem 5.1. Let us consider $1 \leq p \leq \infty$, an open set $\Omega \subseteq \mathbf{R}$ and a $p$-admissible vectorial measure $\mu=\left(\mu_{0}, \ldots, \mu_{k}\right)$ in $\bar{\Omega}$ with $(\bar{\Omega}, \mu) \in \overline{\mathcal{C}}$. Then the Sobolev space $W^{k, p}(\bar{\Omega}, \mu)$ is complete. 
Remark. The hypothesis of $p$-admissibility is natural (see definitions 8 and 9 and their remarks). Also the condition $(\bar{\Omega}, \mu) \in \mathcal{C}$ is not very restrictive (see Definition 11 and its remarks in Section 4).

Proof. Let $\left\{f_{n}\right\}$ be a Cauchy sequence in $W^{k, p}(\bar{\Omega}, \mu)$. Therefore, for each $0 \leq j \leq k,\left\{f_{n}^{(j)}\right\}$ is a Cauchy sequence in $L^{p}\left(\bar{\Omega}, \mu_{j}\right)$ and it converges to a function $g_{j} \in L^{p}\left(\bar{\Omega}, \mu_{j}\right)$.

First of all, let us show that $g_{j}$ can be extended to a function in $C\left(\Omega^{(j)}\right)$ (if $0 \leq j<k$ ) and in $L_{l o c}^{1}\left(\Omega^{(j-1)}\right)$ (if $0<j \leq k$ ).

If $0 \leq j<k$, let us consider any compact interval $K \subseteq \Omega^{(j)}$. Theorem 4.3(b) gives that there exists a representative (independent of $K$ ) of the class of $f_{n} \in W^{k, p}(\bar{\Omega}, \mu)$ (which we also denote by $f_{n}$ ) and a positive constant $c$ such that for every $n, m \in \mathbf{N}$

$$
c\left\|f_{n}^{(j)}-f_{m}^{(j)}\right\|_{L^{\infty}(K)} \leq \sum_{i=0}^{k}\left\|f_{n}^{(i)}-f_{m}^{(i)}\right\|_{L^{p}\left(\bar{\Omega}, \mu_{i}\right)} .
$$

As $\left\{f_{n}^{(j)}\right\} \subseteq C(K)$, there exists a function $h_{j} \in C(K)$ such that

$$
c\left\|f_{n}^{(j)}-h_{j}\right\|_{L^{\infty}(K)} \leq \sum_{i=0}^{k}\left\|f_{n}^{(i)}-g_{i}\right\|_{L^{p}\left(\bar{\Omega}, \mu_{i}\right)} .
$$

Since we can take as $K$ any compact interval contained in $\Omega^{(j)}$, we obtain that the function $h_{j}$ can be extended to $\Omega^{(j)}$ and we have in fact $h_{j} \in C\left(\Omega^{(j)}\right)$. It is obvious that $g_{j}=h_{j}$ in $\Omega^{(j)}$ (except for at most a set of zero $\mu_{j}$-measure), since $f_{n}^{(j)}$ converges to $g_{j}$ in the norm of $L^{p}\left(\bar{\Omega}, \mu_{j}\right)$ and to $h_{j}$ uniformly on each compact interval $K \subseteq \Omega^{(j)}$. Therefore we can assume that $g_{j} \in C\left(\Omega^{(j)}\right)$.

If $0<j \leq k$, let us consider any compact interval $J \subseteq \Omega^{(j-1)}$. Now Corollary 4.3(b) gives

$$
c\left\|f_{n}^{(j)}-f_{m}^{(j)}\right\|_{L^{1}(J)} \leq \sum_{i=0}^{k}\left\|f_{n}^{(i)}-f_{m}^{(i)}\right\|_{L^{p}\left(\bar{\Omega}, \mu_{i}\right)} .
$$

As $\left\{f_{n}^{(j)}\right\} \subseteq L^{1}(J)$, there exists a function $u_{j} \in L^{1}(J)$ such that

$$
c\left\|f_{n}^{(j)}-u_{j}\right\|_{L^{1}(J)} \leq \sum_{i=0}^{k}\left\|f_{n}^{(i)}-g_{i}\right\|_{L^{p}\left(\bar{\Omega}, \mu_{i}\right)} .
$$

Since we can take as $J$ any compact interval contained in $\Omega^{(j-1)}$, we obtain that the function $u_{j}$ can be extended to $\Omega^{(j-1)}$ and we have in fact $u_{j} \in L_{l o c}^{1}\left(\Omega^{(j-1)}\right)$. It is obvious that $g_{j}=u_{j}$ in $\Omega^{(j)}$ (except for at most a set of zero Lebesgue measure), since $f_{n}^{(j)}$ converges to $u_{j}$ in $L_{l o c}^{1}\left(\Omega^{(j)}\right)$ and to $g_{j}$ locally uniformly in $\Omega^{(j)}$. Let us consider a set $A$ which concentrates the mass of $\left(\mu_{j}\right)_{s}$, with $|A|=0$; we can take $u_{j}=g_{j}$ in $A$. We only need to show $u_{j}=g_{j}$ in $\Omega_{j} \backslash\left(\Omega^{(j)} \cup A\right.$ ) (recall that by hypothesis $w_{j}=0$ in $\mathbf{R} \backslash \Omega_{j}$ ), but this is immediate since $w_{j} \in B_{p}\left(\Omega_{j}\right)$ and the convergence in $L^{p}\left(\Omega_{j}, w_{j}\right)$ implies the convergence in $L_{l o c}^{1}\left(\Omega_{j}\right)$ (by Lemma 3.1). Therefore we can assume that $g_{j} \in L_{l o c}^{1}\left(\Omega^{(j-1)}\right)$.

In fact, we have seen that $\left\{f_{n}^{(j)}\right\}$ converges to $g_{j}$ in $L_{l o c}^{\infty}\left(\Omega^{(j)}\right)$ (if $0 \leq j<k$ ) and in $L_{l o c}^{1}\left(\Omega^{(j-1)}\right.$ ) (if $0<j \leq k)$.

Let us see now that $g_{j}^{\prime}=g_{j+1}$ in the interior of $\Omega^{(j)}$ for $0 \leq j<k$. Let us consider a connected component $I$ of int $\left(\Omega^{(j)}\right)$. Given $\varphi \in C_{c}^{\infty}(I)$, let us consider the convex hull $K$ of supp $\varphi$. We have that $K$ is a compact interval contained in $I \subseteq \Omega^{(j)}$. The uniform convergence of $\left\{f_{n}^{(j)}\right\}$ in $K$ and the $L^{1}$ convergence of $\left\{f_{n}^{(j+1)}\right\}$ in $K$ gives that

$$
\int_{K} \varphi^{\prime} g_{j}=\lim _{n \rightarrow \infty} \int_{K} \varphi^{\prime} f_{n}^{(j)}=-\lim _{n \rightarrow \infty} \int_{K} \varphi f_{n}^{(j+1)}=-\int_{K} \varphi g_{j+1} .
$$

Then $g_{j+1}=g_{0}^{(j+1)}$ in $\operatorname{int}\left(\Omega^{(j)}\right)$ and $g_{0}^{(j)} \in A C_{l o c}\left(\operatorname{int}\left(\Omega^{(j)}\right)\right)$ for $0 \leq j<k$. In order to see that $g_{0}^{(j)} \in$ $A C_{\text {loc }}\left(\Omega^{(j)}\right)$, it is enough to recall that $\left(g_{0}^{(j)}\right)^{\prime}=g_{j+1} \in L_{l o c}^{1}\left(\Omega^{(j)}\right)$. 


\section{References.}

[A] Adams, R. A., Sobolev Spaces. Academic Press. 1978.

[APRR] Alvarez, V., Pestana, D., Rodríguez, J.M., Romera, E., Weighted Sobolev spaces on curves, J. Approx. Theory 119 (2002), 41-85.

[CE] Chisholm, R. S., Everitt, W. N., On bounded integral operators in the space of integrable-square functions, Proc. Roy. Soc. Edinb. (A) 69 (1970/71), 199-204.

[CEL] Chisholm, R. S., Everitt, W. N., Littlejohn, L. L., An integral operator with applications, J. Inequal. and Appl. 3 (1999), 245-266.

[D] Davis, P. J., Interpolation and Approximation. Dover. 1975.

[EL] Everitt, W. N., Littlejohn, L. L., The density of polynomials in a weighted Sobolev space, Rendiconti di Matematica, Serie VII, 10 (1990), 835-852.

[ELW1] Everitt, W. N., Littlejohn, L. L., Williams, S. C., Orthogonal polynomials in weighted Sobolev spaces, Lecture Notes in Pure and Applied Mathematics, 117 (1989), Marcel Dekker, 53-72.

[ELW2] Everitt, W. N., Littlejohn, L. L., Williams, S. C., Orthogonal polynomials and approximation in Sobolev spaces, Jour. Comput. Appl. Math. 48 (1993), 69-90.

[GR] García-Cuerva, J., Rubio de Francia, J. L., Weighted norm inequalities and related topics. NorthHolland. 1985.

[H] Hajlasz, P., Sobolev spaces on an arbitrary metric space, Potential Anal. (to appear).

[HLP] Hardy, G., Littlewood, J. E., Pólya, G., Inequalities. Cambridge University Press, 1934.

[HKM] Heinonen, J., Kilpeläinen, T., Martio, O., Nonlinear Potential Theory of degenerate elliptic equations. Oxford Science Publ. Clarendon Press, 1993.

[K] Kilpeläinen, T., Weighted Sobolev spaces and capacity, Ann. Acad. Sci. Fennicae, Series A. I. Math. 19 (1994), 95-113.

[Ku] Kufner, A., Weighted Sobolev Spaces. Teubner Verlagsgesellschaft, Teubner-Texte zur Mathematik (Band 31), 1980. Also published by John Wiley \& Sons, 1985.

[KO] Kufner, A., Opic, B., How to define reasonably Weighted Sobolev Spaces. Commentationes Mathematicae Universitatis Caroline 25(3) 1984, 537-554.

[KS] Kufner, A., Sändig, A. M., Some Applications of Weighted Sobolev Spaces. Teubner Verlagsgesellschaft, Teubner-Texte zur Mathematik (Band 100), 1987.

[LP] López Lagomasino, G., Pijeira, H., Zero location and $n$-th root asymptotics of Sobolev orthogonal polynomials, Jour. Approx. Theory 99 (1999), 30-43.

[LPP] López Lagomasino, G., Pijeira, H., Pérez, I., Sobolev orthogonal polynomials in the complex plane, J. Comp. Appl. Math. 127 (2001), 219-230.

[M] Martio, O., The Hardy-Littlewood maximal function and Sobolev spaces on a metric space. Preprint.

[Ma] Maz’ja, V. G., Sobolev spaces. Springer-Verlag. 1985.

[Mu1] Muckenhoupt, B., Weighted norm inequalities for the Hardy maximal function, Trans. Amer. Math. Soc. 165 (1972), 207-226.

[Mu2] Muckenhoupt, B., Hardy's inequality with weights, Studia Math. 44 (1972), 31-38.

[RARP] Rodríguez, J. M., Alvarez, V., Romera, E. y Pestana, D., Generalized weighted Sobolev spaces and applications to Sobolev orthogonal polynomials II, Approx. Theory and its Appl., 18:2 (2002), 1-32.

[R1] Rodríguez, J. M., Weierstrass' Theorem in weighted Sobolev spaces, J. Approx. Theory 108 (2001), 119-160.

[R2] Rodríguez, J. M., The multiplication operator in weighted Sobolev spaces with respect to measures, J. Approx. Theory 109 (2001), 157-197.

[R3] Rodríguez, J. M., Approximation by polynomials and smooth functions in Sobolev spaces with respect to measures, J. Approx. Theory 120 (2003), 185-216. 
[RY] Rodríguez, J. M., Yakubovich, V.A., A Kolmogorov-Szegö-Krein type condition for weighted Sobolev spaces. Preprint.

[T] Talenti, G, Osservazioni sopra una classe di disuguaglianze, Rend. Sem. Mat. Fis. Milano 39 (1969), 171-185.

[To] Tomaselli, G, A class of inequalities, Boll. Un. Mat. Ital. 4 (1969), 622-631.

[Tr] Triebel H., Interpolation Theory, Function Spaces, Differential Operators. North-Holland Mathematical Library, 1978.

Domingo Pestana, Elena Romera, José M. Rodríguez

Departamento de Matemáticas

Universidad Carlos III de Madrid

Avenida de la Universidad, 30

28911 Leganés (Madrid), SPAIN

dompes@math.uc3m.es, eromera@math.uc3m.es, jomaro@math.uc3m.es
Venancio Alvarez

Departamento de Análisis Matemático

Facultad de Ciencias

Campus de Teatinos

29071 Málaga, SPAIN

nancho@anamat.cie.uma.es 